\title{
Formation and evolution of early-type galaxies: spectro-photometry from cosmo-chemo-dynamical simulations
}

\author{
R. Tantalo ${ }^{1}$, S. Chinellato ${ }^{2}$, E. Merlin ${ }^{1}$, L. Piovan ${ }^{1}$, and C. Chiosi ${ }^{1}$ \\ 1 Department of Astronomy, Padova University, Vicolo dell'Osservatorio 3, 35122 Padova, Italy \\ e-mail: [rosaria.tantalo; emiliano.merlin; cesare.chiosi]@unipd.it;lorenzo.piovan@gmail.com \\ 2 Padova Astronomical Observatory, Vicolo dell'Osservatorio 5, 35122 Padova, Italy \\ e-mail: simonetta.chinellato@oapd.inaf.it
}

Received 17 June 2009 / Accepted 19 April 2010

\begin{abstract}
Context. One of the major challenges in modern astrophysics is to understand the origin and the evolution of galaxies, the bright, massive early type galaxies (ETGs) in particular. There is strong observational evidence that massive ETGs are already in place at redshift $z \sim 2-3$ and that they formed most of their stars well before $z=1$. Therefore, these galaxies are likely to be good probes of galaxy evolution, star formation and, metal enrichment in the early Universe.

Aims. In this context it is very important to set up a diagnostic tool able to combine results from chemo-dynamical $N$-Body-TSPH (NB-TSPH) simulations of ETGs with those of spectro-photometric population synthesis and evolution so that all key properties of galaxies can be investigated. These go from the integrated spectrum and magnitudes in any photometry, both in the rest-frame and as a function of the redshift, to present-day structural properties. The main goal of this paper is to provide a preliminary validation of the software package before applying it to the analysis of observational data.

Methods. The galaxy models in use where calculated by the Padova group in two different cosmological scenarios: the standard cold dark matter cosmology (SCDM), and the so-called Concordance cosmology $\left(\Lambda \mathrm{CDM}\right.$, with $\left.\Omega_{\Lambda}=0.762\right)$. For these template galaxies, we recover their spectro-photometric evolution through the entire history of the Universe. This is done in particular for two important photometric systems, the Bessell-Brett and the Sloan Digital Sky Survey (SDSS) passbands.

Results. We computed magnitudes and colors and their evolution with the redshift along with the evolutionary and cosmological corrections for the model galaxies at our disposal, and compared them with data for ETGs taken from the COSMOS and the GOODS databases. Finally, starting from the dynamical simulations and photometric models at our disposal, we created synthetic images in a given photometric system, from which we derived the structural and morphological parameters. In addition to this, we address the question of the scaling relations, and in particular we examine the one by Kormendy. The theoretical results are compared with observational data of ETGs selected form the SDSS database.

Conclusions. The simulated colors for the different cosmological scenarios follow the general trend shown by galaxies of the COSMOS and GOODS surveys at lower redshifts and are in good agreement with the data up to $z \sim 1$, where the number of early-type galaxies observed falls abruptly. In conclusion, within the redshift range considered, all the simulated colors reproduce the observational data quite well. Looking at the structural parameters derived from the surface imaging, the luminosities and effective radii (Kormendy relation) measured for our model galaxies are consistent with the archival data from the SDSS.
\end{abstract}

Key words. galaxies: evolution - galaxies: formation - galaxies: photometry - galaxies: elliptical and lenticular, cD

\section{Introduction}

The origin and evolution of early-type galaxies (ETGs), the bright massive ETGs in particular, are two of the major challenges in modern astrophysics, and it is still a very controversial subject (Chiosi 2000). Spheroidal systems are of interest in their own right because they contain more than half of the total stellar mass in the local Universe (Fukugita et al. 1998). Giant ETGs appear to define a homogeneous class of objects that predominantly consists of uniformly old and red populations, which implies that they must have formed at high redshift and that they have negligible amounts of gas and very little star formation (Bressan et al. 1994).

There is strong observational evidence that old, massive, red, and metal-rich proto-ETGs are already in place at $z \sim 2-3$ and that the present-day early-type galaxies formed most of their stars well before redshift $z=1$ (Searle et al. 1973; Brinchmann \& Ellis 2000; Treu et al. 2005; van der Wel et al. 2005).
Moreover, the current rates of star formation in these systems are quite low, whereas the rates increase sharply into the past (Butcher \& Oemler 1978; Dressler 1980). Therefore, these ETGs are likely good probes of galaxy assembly, star formation, and metal enrichment in the early Universe.

The cosmological background. In a Universe dominated by cold dark matter (CDM), some kind of dark energy in form of the cosmological constant $\Lambda$, and containing a suitable mix of baryons and photons, the cosmic structures are formed by the gravitational collapse of dark matter and are organized in a hierarchy of halos inside which baryons dissipate their energy and collapse to form luminous systems. The formation of ETGs can be reduced to the following schemes (Peebles 2002; Schade et al. 1999):

i) Early, monolithic-like aggregation. This scenario of galaxy formation predicts that all ETGs form at high redshift $(z \gg 1)$ as 
a result of rapid and dissipation-less collapse of a large mass of gas soon transformed into stars. In the model, first proposed by Eggen et al. (1962) and then refined and improved by Larson (1975), Arimoto \& Yoshii (1987), Bressan et al. (1994), and Chiosi \& Carraro (2002), ETGs undergo a single and short, but intense, burst of star formation, followed ever since by the passive evolution of their stellar populations to the present day. This simple model naturally accounts for the old ages ( $\sim 12 \mathrm{Gyr})$ of spheroidal galaxies, their high densities, and the weak temporal evolution of their stellar content.

In favor of this scheme are the observational data that convincingly hint at old and homogeneous stellar populations (see Chiosi 2000, for a review of the subject). It is worth mentioning, however, that Kauffmann et al. (1993) and Barger et al. (1999) argue for some recent evolution in the stellar populations of elliptical galaxies. This scenario reproduces the optical properties of ETGs remarkably well, and successfully explains the tightness of the fundamental scaling relations that ETGs obey, like the color-magnitude relation and the fundamental plane, as well as the evolution of these relations as a function of redshift.

The monolithic formation mechanism fails to explain some recent observational evidence that has become available with the advance of more detailed data from present-day surveys. These indicate that the star formation histories of at least some ETGs, and perhaps the early-type population as a whole, deviate strongly from the expectations of the monolithic collapse paradigm, both in terms of their structural evolution and star formation experienced by them over the whole Hubble time. It is less successful at explaining the detailed luminosity dependence of their dynamical properties, the apparent scarcity of very large star-bursts in the high-redshift universe, and the origin of dynamical peculiarities indicating some recent accretion events. This scenario does not fit the currently accepted $\Lambda$ CDM picture of galaxy formation whose bottom line is that massive dark matter halos are assembled by mergers of low-mass halos and therefore the mass of a galaxy is thought to accumulate over the lifetime of the Universe.

To cope with some of the above difficulties, a hybrid scenario named revised monolithic has been proposed by Schade et al. (1999) and confirmed by NB-TSPH simulations by Merlin \& Chiosi (2006, 2007), who suggest that a large number of the stars in massive galaxies are formed very early-on at high redshift $(z \sim 1-2)$ and the remaining few at lower $z$. The revised monolithic ought to be preferred to the classical monolithic, as some evidence of star formation at $0.2 \leq z \leq 2$ can be inferred from the emission line of [OII], and also the number frequency of ETGs up to $z \simeq 1$ seems to be nearly constant. Recently, Pérez-González et al. (2008), whom analyzing a huge sample of galaxies, confirmed a scenario where most massive objects assemble their mass very early, whereas the smallest galaxies evolve more slowly building up their mass at lower redshift.

ii) The hierarchical aggregation. This scenario instead suggests that massive ETGs are the end product of subsequent violent mergers of preexisting smaller subunits, on time scales almost equal to the Hubble. In this scenario, the epoch of assembly of ETGs differs markedly from the epoch of formation of their constituent stars, and the high density of elliptical galaxies is ascribed to the effects of dissipation during the formation of the progenitor disks. As the look-back time increases, the density in comoving space of bright (massive) ETGs should decrease by a factor 2 to 3 (see e.g. White \& Rees 1978; Kauffmann et al. 1993).

This model accounts naturally for the scarcity of very bright elliptical progenitors at high redshift, for the rapid evolution of the galaxy population with look-back time, and for dynamical peculiarities. In favor of this view is some observational evidence that the merger rate likely increases with $\sim(1+z)^{3}$ (Patton et al. 1997), together with some hint for a color-structure relationship for E \& S0 galaxies: the color becomes bluer at increasing complexity of a galaxy structure. This could indicate some star formation associated to the merger event. Finally, there are the many successful numerical simulations of galaxy encounters, mergers, and interactions (e.g. Barnes \& Hernquist 1996).

It is, on the other hand, less successful in explaining the apparent old ages of stars in elliptical galaxies and their uniformity in dynamical properties. Nevertheless, contrary to the expectation from this model, the number density of ellipticals do not seem to decrease with the redshift, at least up to $z \simeq 1$ (Im et al. 1996). A significant population of massive and passive ETGs up to $z \approx 2.5$ and some hints about massive ETGs at redshift $z>3$ (Cimatti 2009), clearly do not agree with the classical hierarchical scenario, because we need to have big objects already in place at higher and higher $z$.

There is a companion scheme named dry merger, in which bright ETGs form by encounters of quiescent, no star-forming galaxies. This view is advocated by Bell et al. (2004), who find that the $B$-band luminosity density of the red peak in the color distribution of galaxies shows mild evolution starting from $z \simeq 1$. As old stellar populations would fade by a factor 2 or 3 in this time interval, and the red color of the peak tells us that new stars are not being formed in old galaxies, this mild evolution hints for a growth in the stellar mass of the red sequence, either coming from the blue-peak galaxies in which star formation is truncated by some physical process, or by "dry mergers" of smaller red, gas-poor galaxies. However, according to Bundy et al. (2005, 2006), dry mergers cannot be the leading mechanism in the history of galaxy assembly because of the weak dependence on the environment, in contrast to what expected. Indeed the majority of quiescent galaxies seem to be assembled by a mechanisms that depends on their mass rather than the environment, as the merger rate does not seem to increase with environment density (Bundy et al. 2006).

Putting Dynamics and Photometry together. How can we disentangle the above scenarios? Comparing theoretical predictions to observational data concerning the light and hence mass profiles, velocity, SEDs, magnitudes, colors, line strength indices, and associated gradients. In this context, spectro-photometric models of galaxies have long been the key tool to investigate how galaxies formed and evolved with time. Consequently, an impressive number of chemo-spectro-photometric models for ETGs have been proposed. To mention a few among the recent ones, we recall Bressan et al. (1994), Vazdekis et al. (1997), Tantalo et al. (1996), Gibson (1997), Kodama \& Arimoto (1997), Fioc \& Rocca-Volmerange (1997), Tantalo et al. (1998b), Pipino \& Matteucci (2004), and Piovan et al. (2006a,b). Nearly all these models simulate a galaxy and its evolution adopting the point source approximation, in which no morphological structure and no dynamics are considered.

In parallel to this line of work, many fluid-dynamical models in $n$-dimensions (from 1 to 3 ) and with multi-phase descriptions of the gaseous component were developed for galaxies of different morphological type. To mention a few, we recall Theis et al. (1992), Ferrini \& Poggianti (1993), Samland et al. (1997), Boissier \& Prantzos (1999b,a), Samland \& Gerhard (2000), Samland (2001), Berczik et al. (2003), and Immeli et al. (2004). For some of them the spectro-photometric aspect of the models was also investigated with successful results. 
Finally, there are the NB-TSPH simulations with dark and baryonic matter, the hydrodynamic treatment of the baryonic component, and even multi-phase descriptions of the gas. The NB-TSPH simulations are one of the best tools to infer the 3D structure of ETGs, to follow the temporal evolution of the dynamical structure, the stellar content, and the chemical elements. Recent models of this type at different level of complexity are by Chiosi \& Carraro (2002), Kobayashi (2004b,a, 2005), Merlin \& Chiosi (2006, 2007), and Scannapieco et al. (2006b,a). So far the corresponding photometric properties of the models are either left aside or treated in a very rudimentary way.

It follows from these considerations that the ideal tool to develop would be the one folding together NB-TSPH simulations and chemo-spectro-photometry to generate 3D chemodynamical, spectro-photometric models of galaxies. This would allow us to simultaneously predict and discuss both the structural properties related to dynamical formation process and the spectro-photometric ones related to the stellar content in a self consistent fashion, and hopefully to cast light on the above issues. Therefore, we have taken the cosmo-chemo-dynamical models of galaxies calculated with the Padova Code [GALDYN] (see details below) from which we get the star formation (SFH), the chemical enrichment $(Z(t))$ histories, and the structure of the simulated galaxy. The output of these models is fed into the Padova photometric code [SPECODY], which generates the spectral energy distribution (SED) of the whole galaxy. From this SED we derive the absolute magnitudes, colors, indices, etc., in a chosen photometric system. This allows us to determine the rest-frame and cosmological evolution of magnitudes and colors for the set of models at our disposal.

Aims and plan of the paper. The purpose of this study is to validate the whole procedure before applying it to a set of simulations under preparation and/or to extensive study of observational data. The outline of the paper is as follows. Section 2 describes the dynamical NB-TSPH simulations of ETGs. Section 3 describe the photometric package used to get the rest-frame magnitudes and colors of the galaxy models in a photometric system (some details are given for two of those, namely the Bessell-Brett and the Sloan Digital Sky Survey (SDSS). We also we present a study of the color-magnitude diagram (CMD) of the stellar populations of the model galaxies. Section 4 presents a multi-wavelength study of the optical and near-IR high- $z$ photometric properties of the ETGs and compares the results with a sample of galaxies selected from COSMOS and GOODS surveys. In Sect. 5 we describe the method followed to derive $2 \mathrm{D}$ artificial images, starting from the $3 \mathrm{D}$ model galaxies. These images resemble observational data and can be analyzed in a similar manner. Isophotal analysis with aid of the Fourier and Sérsic technique is applied to derive some structural properties of the model galaxies. We obtain the morphological and structural parameters and compare them with the data for a sample of elliptical galaxies selected from SDSS. This allows us to establish the consistency of the models with photometric data. In Sect. 6 we use the parameters derived from the surface photometry to investigate the Kormendy scaling relation. Finally, in Sect. 7, we discuss some unsettled issues that require future work and present some general, conclusive remarks.

\section{Dynamical models of ETGs}

For our analysis we have considered three numerical simulations, calculated by Merlin \& Chiosi (2006, 2007) using
[GALDYN] the cosmo-chemo-dynamical evolutionary code developed by Merlin \& Chiosi $(2006,2007)$. The code stems from the original NB-TSPH code developed in Padova by Carraro et al. (1998). It combines the Oct-Tree algorithm (Barnes \& Hut 1986) for the computation of the gravitational forces with the SPH (Lucy 1977; Benz 1990) approach to numerical hydrodynamics of the gas component. It is fully-Lagrangian, threedimensional, and highly adaptive in space and time owing to individual smoothing lengths and individual time-steps. It includes self-consistently a number of non-standard physical processes: radiative and inverse Compton cooling, star formation, energy feedback, and metal enrichment by type Ia and II SNæ (Lia et al. 2002). The numerical recipe for star formation, feedback, and chemical enrichment along with all the other physical processes considered, the improvements to the initial conditions and the multi-phase description of the interstellar medium are described in Merlin \& Chiosi (2006, 2007). No details are given here but for a few key points.

Particles, representing dark matter and baryons both in form of gas and stars, evolve in the dynamical phase space under the action of cosmological expansion, self-gravity, and (in the case of gas) hydrodynamical forces. In the single-phase description (only one type of gas), the gas-particles are turned into starparticles as soon as they satisfy three physical requirements: (i) to be denser than a threshold value; (ii) to belong to a convergent flow; and (iii) to cool efficiently. There is also an additional statistical criterium (as described in details in Lia et al. 2002) to be fulfilled. In the multi-phase description (hot-rarefied and cool-dense gas), gas-particles that become colder and denser than suitable thresholds are subtracted from the SPH scheme and turned into sticky particles; in this case, star formation can take place only within this cold and dense phase. Star-particles then refuel the interstellar medium with energetic and chemical feedbacks, ultimately quenching star formation when the gas heated by $\mathrm{SN}$ explosions is hot enough to leave the galaxy potential well (galactic winds).

Two cosmological scenarios are adopted to calculate the galaxy models: the so-called standard-CDM (SCDM) and the concordance $\Lambda \mathrm{CDM}$ as inferred by WMAP3 data (Spergel et al. 2003). One galaxy model is calculated with SCDM and the onephase description, and two with $\Lambda$ CDM. These latter in turn differ for the treatment of the interstellar medium: (i) one-phase medium; the model is shortly indicated as $\Lambda \mathrm{CDM}$ and (ii) multiphase medium; the models is named $\Lambda \mathrm{CDM}_{\mathrm{mp}}$ (see Merlin \& Chiosi 2006, 2007, and below for more details).

All the models are constructed as follows: we start from a realistic simulation of a large region of the Primordial Universe carried out with a given cosmological scenario. At certain value of the redshift, typically $z_{\text {ini }} \simeq 50-60$ (the precise value changes from model to model), a spherical, over-dense, galaxy-sized proto-halo is selected, detached from its surroundings, and let evolve with void boundary conditions, after that an outwards radial initial velocity has been added to simulate the Hubble flow. Initially, the proto-halo continues to expand but, reached a maximum extension, it turns around and collapses toward higher and higher densities. In the meantime, baryons (gas) collapse too and start forming stars, at the beginning very slowly and then at increasing rate. The redshift at which significant star formation begins is in between 50-60 and 5, but close to about 5. In all models at redshift about 2 the conversion of gas into stars is nearly complete Merlin \& Chiosi (see 2006, 2007, for all details). In this picture, there is no sharp value of the redshift at which star formation is supposed to start, $z_{\text {ini }}$ is simply the redshift at which the proto-halo, inside which a galaxy will later be formed, is 
Table 1. Cosmological parameters adopted in our simulations.

\begin{tabular}{cccc}
\hline \hline Model & SCDM & $\Lambda$ CDM & $\Lambda$ CDM $_{\mathrm{mp}}$ \\
\hline$h_{0}$ & 0.5 & 0.730 & 0.730 \\
$\Omega_{\mathrm{M}}$ & 1 & 0.238 & 0.238 \\
$\Omega_{\Lambda}$ & 0 & 0.762 & 0.762 \\
$\sigma_{8}$ & 0.5 & 0.740 & 0.740 \\
\hline
\end{tabular}

singled out from the cosmological tissue. For a similar choice of $z_{\text {ini }}$ see also Li et al. (2006b,a,c, 2007). Table 1 provides a summary of the relevant cosmological parameters.

Inside each proto-halo (proto-galaxy), the baryonic component is initially in the gaseous phase and follows the dark matter perturbations until it is heated up by shocks and mechanical friction. When radiative cooling becomes efficient, the first cold clumps begin to form, and the gas is finally turned into starparticles. Because of the mass resolution of the models, a starparticle is so massive that it can be thought of to correspond to an assembly of real stars, which in turn distribute in mass according to some initial mass function over the mass interval $m_{1}$ to $m_{\mathrm{u}}$, i.e. 0.1 to $100 M_{\odot}$. At the present time, each star-particle contains living stars, from $m_{1}$ to a maximum mass $m_{\max }(t)$ that depends on the age, and remnants (black holes, neutron stars, and white dwarfs) generated by all stars in the mass interval $m_{\max }(t)<m<m_{\mathrm{u}}$. Therefore, in a star-particle, SN-explosions may occur (their rate can be easily calculated), thus releasing energy that cause evaporation of the nearby clouds which quenches the star formation.

The three galaxy models we have considered differ in important aspects that deserve some comments:

i) For the SCDM model (standard cold dark matter cosmology) calculated with the one-phase description of the interstellar medium, the cosmological parameters are chosen in accordance with the kind of model that cosmologists classify as the reference case. This explains why we have chosen the normalized Hubble constant $h_{0}=0.5$ although nowadays $h_{0}=0.7$ ought to be preferred. Nevertheless, since testing cosmology is beyond the scope of this study which simply aims to test the ability of our code in predicting the photometric properties of the model galaxy, the exact choice of $h_{0}$ is not particularly relevant here.

ii) The models $\Lambda C D M$ and $\Lambda \mathrm{CDM}_{\mathrm{mp}}$ refer to the standard concordance cosmology in presence of dark energy. However, they have different assumptions concerning the treatment of the interstellar medium: only one phase for the first and two phases for the second.

iii) As a consequence of the different cosmological backgrounds, the three models do not have the same initial total mass nor the same ratio $f_{\mathrm{B}}$ of baryonic to total mass, nor the initial redshift at with the perturbations are singled out from the cosmological tissue.

iv) The evolution of the model galaxies is followed up to the present, except for the $\Lambda \mathrm{CDM}$ simulation that stops at $z \sim 1$ and age of about $7 \mathrm{Gyr}$ in the adopted cosmology, due to computational difficulties. However, for the purposes of this paper, to consider also this truncated model is safe. Indeed, the model has already relaxed to dynamical equilibrium so that its shape will not change significantly during the remaining 5 Gyr. The rate of star formation has already decreased to very low levels like in SCDM and $\Lambda \mathrm{CDM}_{\mathrm{mp}}$ models, and there is no reason to imagine that it would strongly increase during the age interval from 7 to 13 Gyr. From a photometric point of view, it is a galaxy in passive evolution.

v) The numerical simulations track the metal content of each gas- and star-particle. In brief, using the prescription for chemical evolution by Lia et al. (2002), in each gas- and star-particle the evolution of the mass abundance of 10 elements ( $\mathrm{He}, \mathrm{C}, \mathrm{O}, \mathrm{N}, \mathrm{Mg}, \mathrm{Si}, \mathrm{S}, \mathrm{Ca}$, and $\mathrm{Fe}$ ) and the total metallicity $Z$ is followed in detail. Each star-particle carries its own age and chemical composition "tag". It is worth noting that the pattern of abundances of the galaxy models is fully consistent with the ones adopted to calculate the evolutionary tracks and isochrones at the base of our SSPs.

vi) To conclude, all the three models can be used for the aims of this study, i.e. to set up the photometric package suited to NB-TSPH simulations and to validate it.

In Table 2 we summarize the initial dynamical and computational parameters for the three models. The total mass $M_{\text {tot }}$ of the model galaxies within the initial sphere radius $R$ comprises both the dark $M_{\mathrm{DM}}$ and baryonic matter $M_{\mathrm{B}}$, in the cosmological ratio $f_{\mathrm{B}}$. We also list the initial total number of DM- and B-particles, these latter in form of gas, together with the mass value per particle shortly indicated as $M_{\mathrm{DM}, \mathrm{p}}$ and $M_{\mathrm{B}, \mathrm{p}}$. The mass per particle depends on the number of particles adopted for each species. In the course of evolution, the gas-particles are turned into star-particles of the same mass, which in the text is indicated as $m_{\mathrm{sp}}$. We give the softening length for DM- and B-particles that is used to calculate the gravitational interaction. In brief, because of the time and mass resolution limits, close encounters between particles may cause numerical errors. To cope with this, it is common practice to soften the gravitational force between close pairs of bodies: if the distance between them becomes smaller than a suitable softening length $\epsilon$, the force exerted on each body is corrected and progressively reduced to zero with decreasing distance. Finally, we give the IMF that is used to calculate chemical enrichment and energy feedback by the real stars composing each star-particle (see below for more details).

\subsection{Results for ETG models}

The final properties of the three galaxies considered are summarized in Table 3 (for other parameters regarding the simulations see Merlin \& Chiosi 2006, 2007).

Figure 1 shows the star formation history ( $\mathrm{SFH}$ ) versus time, in Gyr, and/or redshift for the three models. Stars form in clumps of cold gas that have collapsed on small scales so that the entire process can be described as triggered by a number of early dissipative gravitational collapses, followed by very early merging of stellar substructures. As it is clearly shown, the galaxies form from an initial star-burst comprised in the time interval 1 to 3 Gyr in all cases. As the cold gas is depleted, the SFR declines rapidly. Anyway, at lower redshifts small amounts of the previously heated gas have cooled down again at the center of the galaxy, so that SFR may continue till the present epoch $(z=0)$, even if at much lower rates. Although we suspect that to a great extent, this feature of the models might be of numerical nature, there are no strong compelling physical reasons to rule it out. Some residual star formation could occur even at the present time in the very central regions of ETGs. If so, some effects on the central colors are easy to foresee (see below).

Figure 2 displays the stellar mass assembly process, i.e. the growth with time of the total mass in form of stars, $M_{\text {star. }}$ The evolution turns the initial irregular proto-galaxy into a well shaped spheroid, that quickly relaxes into the final configuration, 
R. Tantalo et al.: Spectro-photometric models of early type galaxies

Table 2. Initial dynamical and computational parameters for the three model galaxies.

\begin{tabular}{lrccc}
\hline \hline \multicolumn{1}{c}{ Model } & & SCDM & $\Lambda$ CDM & $\Lambda$ CDM \\
\hline Initial Total Mass [ $M_{\odot}$ ] & $M_{\text {tot }}$ & $1.625 \times 10^{12}$ & $8.830 \times 10^{11}$ & $2.449 \times 10^{11}$ \\
Initial Baryonic Mass Fraction & $f_{\mathrm{B}}$ & 0.1 & 0.176 & 0.176 \\
Initial Baryonic Mass [ $M_{\odot}$ ] & $M_{\mathrm{B}}$ & $1.624 \times 10^{11}$ & $1.413 \times 10^{11}$ & $4.164 \times 10^{10}$ \\
Initial Sphere Radius [kpc] & $R$ & 33.2 & 28 & 19 \\
Initial Number of Dark Matter Particles & & 13685 & 13657 & 6966 \\
Initial Number of Gas Particles & & 13719 & 13707 & 6995 \\
Dark Mass per particle [ $M_{\odot}$ ] & $M_{\mathrm{DM}, \mathrm{p}}$ & $1.069 \times 10^{8}$ & $5.435 \times 10^{7}$ & $2.919 \times 10^{7}$ \\
Baryonic Mass per particle [ $\left.M_{\odot}\right]$ & $M_{\mathrm{B}, \mathrm{p}}$ & $1.184 \times 10^{7}$ & $1.031 \times 10^{7}$ & $5.954 \times 10^{6}$ \\
Initial Redshift of the Proto-Halo & $z_{\text {ini }}$ & 50 & 60 & 58 \\
IMF & & Kroupa & Kroupa & Salpeter \\
Softening length for Dark Matter [kpc] & $\epsilon_{\mathrm{DM}}$ & 2 & 2 & 2 \\
Softening length for Baryonic Matter [kpc] & $\epsilon_{\mathrm{b}}$ & 1 & 1 & 1 \\
\hline
\end{tabular}

Table 3. End-product for the three model galaxies.

\begin{tabular}{lcccc}
\hline \hline \multicolumn{1}{c}{ Model } & & SCDM & $\Lambda$ CDM & $\Lambda \mathrm{CDM}_{\mathrm{mp}}$ \\
\hline Final Redshift & & 0 & 0.95 & 0 \\
Age of the last model [Gyr] & $T_{\text {gal }}$ & 13 & 7 & 13 \\
Final Number of Star Particles & & 7674 & 8700 & 5197 \\
Final Star Mass [ $\left.M_{\odot}\right]$ & $M_{\text {star,tot }}^{f}$ & $9.1 \times 10^{10}$ & $8.9 \times 10^{10}$ & $3.09 \times 10^{10}$ \\
Final Gas Mass [ $\left.M_{\odot}\right]$ & $M_{\text {gas,tot }}^{f}$ & $7.2 \times 10^{10}$ & $5.1 \times 10^{10}$ & $1.05 \times 10^{10}$ \\
Final Effective Radius of Stars [kpc] & $R_{\mathrm{e}}$ & 7 & 3 & 4.6 \\
\hline
\end{tabular}
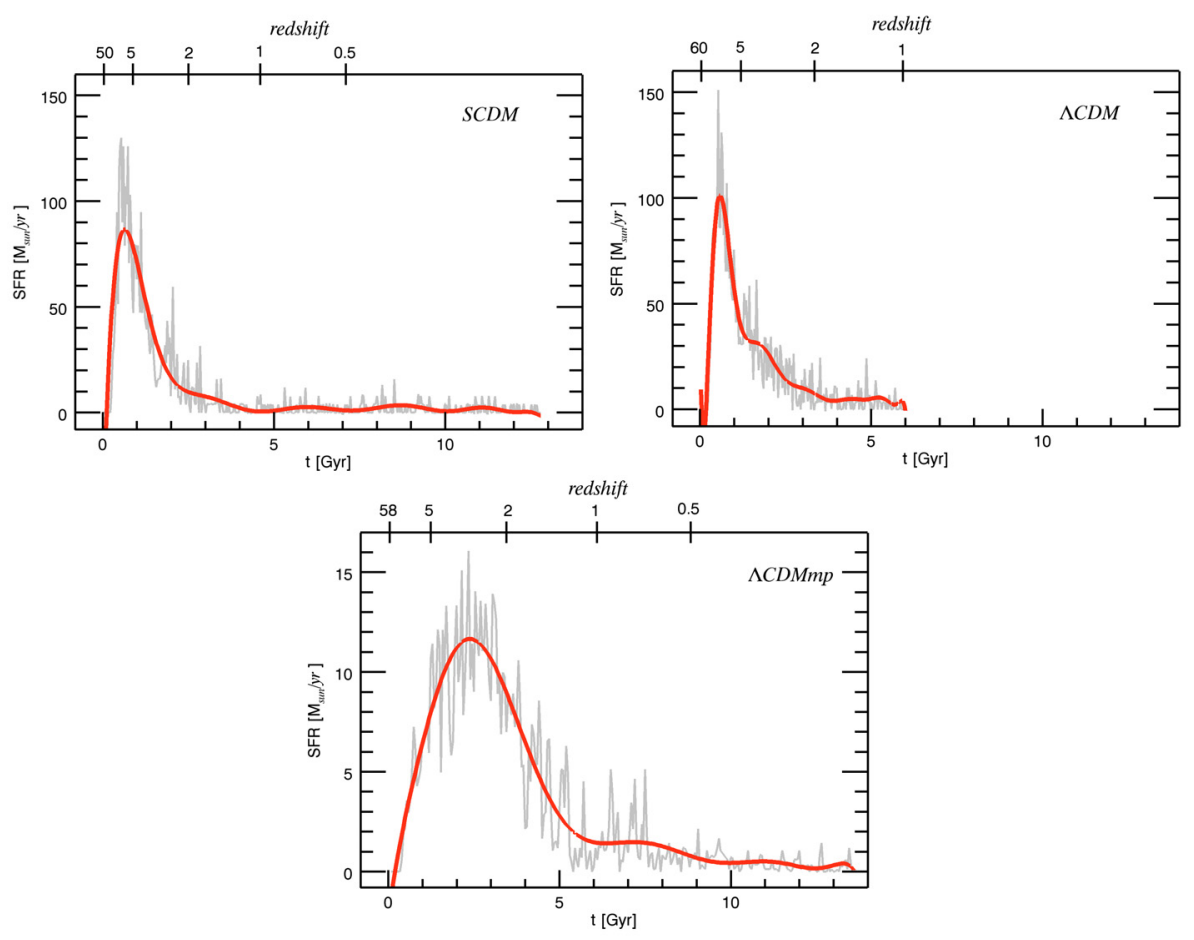

Fig. 1. Star formation rate, in $M_{\odot} / \mathrm{yr}$, versus age, in Gyr, and/or redshift for the different model galaxies considered. Star formation is let start when the proto-galaxy gets gets into existence with respect to the surrounding medium, i.e. $z_{\mathrm{ini}} \sim 50$ for the SCDM model (upper left), $z_{\text {ini }} \sim 60$ for the $\Lambda$ CDM model (upper right), $z_{\text {ini }} \sim 58$ for the $\Lambda \mathrm{CDM}_{\mathrm{mp}}$ model (lower).

closely resembling a real ETG. The final stellar mass is essentially fixed at $z \sim 2$, since little gaseous material is added to the galaxy afterwards and stars age passively for the remaining time of the evolution. Due to the early star-burst, the galaxy is already old and very massive at a redshift of $z \sim 1-2$ for the different models.

Finally, in Fig. 3 we show the mean metallicity versus age relationship for the three models (left panel). The mean metallicity is simply the mean value of all star-particles evaluated at different ages. The right panel shows the metallicity distribution (number of star-particles per metallicity bin) in the three models. The histograms labelled SCDM and $\Lambda \mathrm{CDM}_{\mathrm{mp}}$ refer to the 13 Gyr age models, whereas the $\Lambda$ CDM one is for the 7 Gyr age model. It is worth noting the long tail towards high metallicities. The stars with these high metallicities are responsible of a great deal of the ultraviolet excess in the SEDs via the so-called AGB manqué phase. The same consideration applies to the very old stars in the lowest metallicity bin which may contribute to the ultraviolet excess via the extended horizontal branch phase (see below). 

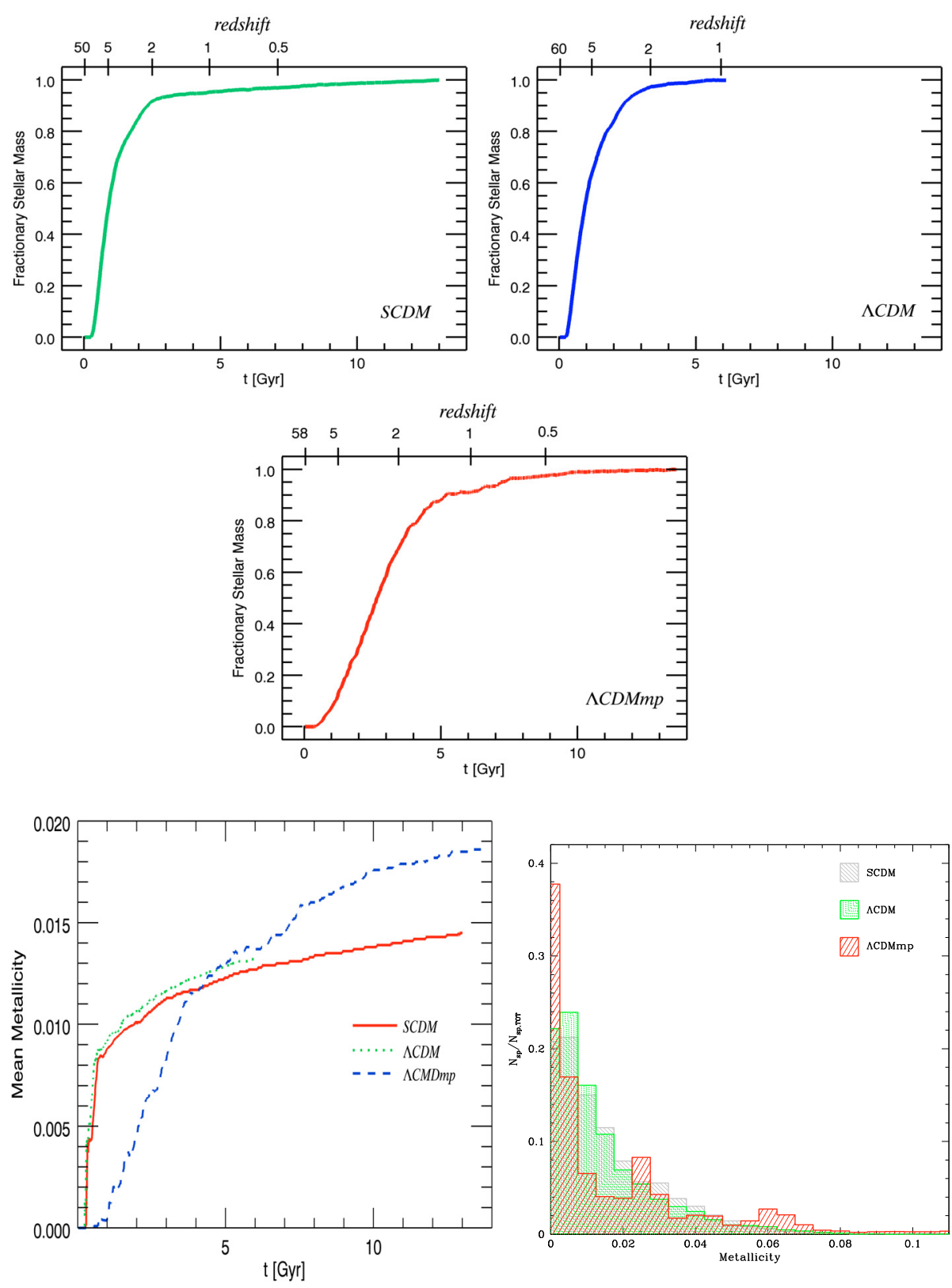

Fig. 2. Growth of the fractionary total star mass $M_{\text {star }}(t) / M_{\text {star }}^{f}$ as a function of the age, in Gyr, and/or redshift for the different models. The final value of $M_{\text {star }}^{f}$ is the quantity given in Table 3.

Fig. 3. Left panel: mean metallicity $Z(t)$ versus time (in Gyr) for the three galaxy models, as indicated. Right panel: metallicity distribution (number of star-particles per metallicity bin) in the three models. The histograms labelled SCDM and $\Lambda \mathrm{CDM}_{\mathrm{mp}}$ refer to the $13 \mathrm{Gyr}$ age model, whereas the $\Lambda \mathrm{CDM}$ one is for the 7 Gyr age model.

\section{The spectral energy distribution of a NB-TSPH simulation: SPECODY}

As already mentioned, owing to the mass resolution of the dynamical simulations fixed by the number of particles to our disposal, each star-particle has the mass $M_{\mathrm{sp}} \sim 10^{7} M_{\odot}$ or so (see Table 2), i.e. each star-particle represents a big assembly of real stars which distribute in mass according to a given initial mass function and are all born in a short burst of star formation, therefore being homogeneous both in age and chemical composition. In this way, each star-particle can be approximated to a SSP of mass $M_{\mathrm{sp}}$.

To derive the SED of our NB-TSPH galaxy model we start from the definition of the integrated monochromatic flux generated by the stellar content of a galaxy of age $T$

$F_{\lambda}(T)=\int_{0}^{T} \int_{m_{l}}^{m_{u}} S(m, t, Z) f_{\lambda}\left(m, \tau^{\prime}, Z\right) \mathrm{d} t \mathrm{~d} m$

where $S(m, t, Z)$ denotes the stellar birth-rate and $f_{\lambda}\left(m, \tau^{\prime}, Z\right)$ the monochromatic flux of a real star of mass $m$, metallicity $Z$, and age $\tau^{\prime}=T-t$. Separating $S(m, t, Z)$ into the product of the star formation rate $\Psi(t, Z)$ (expressed in suitable units) and the initial mass function $\phi(m)$, the above integral becomes

$F_{\lambda}(T)=\int_{0}^{T} \Psi(t, Z) f_{\mathrm{ssp}, \lambda}\left(\tau^{\prime}, Z\right) \mathrm{d} t$

where

$f_{\mathrm{ssp}, \lambda}\left(\tau^{\prime}, Z\right)=\int_{m_{l}}^{m_{u}} \phi(m) f_{\lambda}\left(m, \tau^{\prime}, Z\right) \mathrm{d} m$

is defined as the integrated monochromatic flux of a SSP, i.e. of a coeval, chemically homogeneous assembly of stars with age $\tau^{\prime}$ and metallicity $Z$. The lower and upper mass limits of integration in equations $m_{1}$ and $m_{\mathrm{u}}$ respectively, define the mass range within which stars are generated by each event of star formation. Therefore, the stellar content of a galaxy can be modelled as the convolution of many SSPs of different composition and age, each of which is weighted by the rate of star formation at the age at which it was born. Extensive tabulations of SSPs at 
varying age, chemical composition, and initial mass function, containing magnitudes and colors for many photometric systems are currently to our disposal in literature (see below for a very short summary of the situation). Conventionally, the SED and monochromatic flux, and hence luminosities in whatsoever passband are calculated assuming that the SSP has a total mass equal to $1 M_{\odot}$. Having established the correspondence between the star-particles of the NB-TSPH simulation with the classical SSP and the consistency between the chemical parameters and IMF of the two descriptions, the monochromatic flux and/or luminosity of a galaxy with age $T$ is given by

$F_{\lambda}(T)=\sum_{i} f_{\mathrm{ssp}, \lambda}\left(\tau_{i}, Z_{i}\right) \times M_{\mathrm{sp}, i}$

where $f_{\mathrm{ssp}, \lambda}\left(\tau_{i}, Z_{i}\right)$ is the monochromatic flux of a SSP of age $\tau_{i}$ and metallicity $Z_{i}$. To get the real monochromatic flux emitted by a star-particle we must multiply $f_{\mathrm{ssp}, \lambda}\left(\tau_{i}, Z_{i}\right)$ by $M_{\mathrm{sp}}$ in solar masses. Finally, the summation extends over all star-particles. Extending the same procedure to the whole wavelength interval we get the multi-wavelength SED, from which we can immediately derive magnitudes and colors.

\subsection{Some details on the SSPs in use}

In this paper we have adopted the SSPs computed by Tantalo (2005) and available online from the Padova Galaxies and Single Stellar Population Models database (GALADRIEL) at http: // www . astro.unipd.it/galadriel/.

The spectral energy distributions (SEDs) of the SSPs have been calculated following the method described by Bressan et al. (1994). To this purpose we have adopted the stellar tracks calculated by Girardi et al. (2000). These stellar models include modern physical input as far as opacity, nuclear reaction rates, neutrino losses, mixing schemes, etc. are concerned. The evolutionary sequences go from the zero age main sequence to the latest evolutionary phases and cover wide ranges of stellar masses and chemical compositions. In particular, they include the planetary nebula phase, the so-called AGB manqué phase that may develop in low-mass stars when the metallicity is higher than about three times solar, and the extended horizontal branch typical of low-mass stars with very low metallicity. Finally, the underlying isochrones are calculated by means of the algorithm of "equivalent evolutionary points" described in Bertelli et al. (1994).

In order to derive SEDs, magnitudes, and colors, corresponding to a source of given luminosity, effective temperature, gravity, and chemical composition, one needs a library of stellar spectra as function of these parameters. The spectral library considered in this paper was assembled by Girardi et al. (2002) adopting the ATLAS9 release (Kurucz 1993) of synthetic atmospheres: these latter are those for the no-overshooting case calculated by Castelli et al. (1997) and subsequently extended by other authors.

For each SSP, GALADRIEL provides also large tabulations of magnitudes and colors for the following photometric systems:

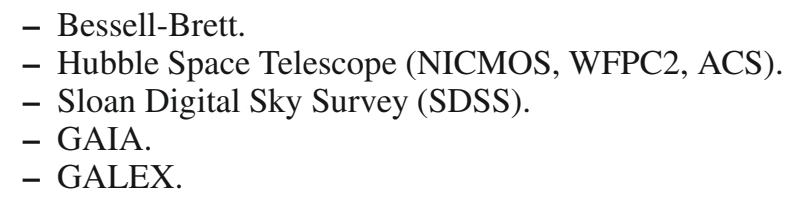

All the details of the computational procedure adopted to derive the synthetic magnitudes and colors are described in Girardi et al. (2002) to whom we refer.
In this study we have considered only two photometric systems: the Bessell-Brett and SDSS for the VEGAmag and ABmag. The transmission curves considered for first photometric system are from Bessell (1990) for the UBVRI passbands and from Bessell \& Brett (1988) for the JHKLMN passbands. The SDSS photometric system (Fukugita et al. 1996) comprises five non-overlapping passbands that range from the ultraviolet cutoff at $3000 \AA$ to the sensitivity limit of silicon CCDs at $11000 \AA$. To interpret large samples of galaxies of recent acquisition, such as COSMOS and GOODS, we have also implemented the photometric systems used in these campaigns (see below).

\subsection{SEDs of the galaxy models}

Using the above technique and integrating over the SEDs of all star-particles we can derive the SEDs of the model galaxies. The SEDs are shown in Fig. 4 at different ages (in view of the cosmological application of these results we remind the reader that these are the SEDs seen in the rest-frame).

It is worth noting that starting from the age of about $5 \mathrm{Gyr}$ the three SEDs show an important ultraviolet excess, i.e. rising branch and a peak in the flux short-ward of $2000 \AA$. At the last age in common, $7 \mathrm{Gyr}$, the flux level is nearly comparable in the SCDM and $\triangle \mathrm{CDM}$ models and significantly lower in the $\Lambda \mathrm{CDM}_{\mathrm{mp}}$ case. For the two models arriving to $13 \mathrm{Gyr}$ age, namely SCDM and $\Lambda C D M_{m p}$, the ultraviolet excess in the first model is significantly higher than in the second one. What is the source of this excess of flux? There are several candidates: the short-lived planetary nebulae, the AGB manqué phase for stars with the appropriate metallicity, the hot horizontal branch stars of low metal content, and finally massive stars if star formation is going on. The Planetary Nebulae are the descendants of low- and intermediate-mass stars on their way from AGB to the White Dwarf regime. Although they can be very bright and hot, they are too short-lived (a few $10^{4} \mathrm{yr}$ ). The AGB manqué stars have a low-mass and a lifetime amounting to a fraction of the core He-burning phase. These stars appear when the age is older than approximately 5-6 Gyr. Low-mass stars of very low metal content during part of their core He-burning phase in a very extended horizontal branch are bright and long lived so that they may significantly contribute to the UV flux. Finally there are the young stars if star formation goes on even at minimal levels of activity. Owing to the much higher intrinsic luminosity of these stars, if they are present even in small numbers they would significantly contribute to the flux in the far ultraviolet. Disentangling the contribution of each possible source is a cumbersome affair. The fact that the flux in this wavelength interval increases starting from about 5 Gyr suggests a combination of AGB manqué stars and young stars, leaving planetary nebulae and extreme horizontal branch objects in the background.

\subsection{Magnitudes and colors of galaxy models}

Given a photometric system, it is straightforward to derive the temporal evolution of magnitudes and colors of each star-particle and of the whole galaxy. For the sake of illustration we show results only for the Bessell-Brett and the SDSS photometric systems.

Star-particle by star-particle view. In Fig. 5 we show the color evolution of the SCDM model galaxy for the Bessell-Brett system. The color code for each star-particle in the 3D-view of the galaxy structure indicates the color evolution of the 

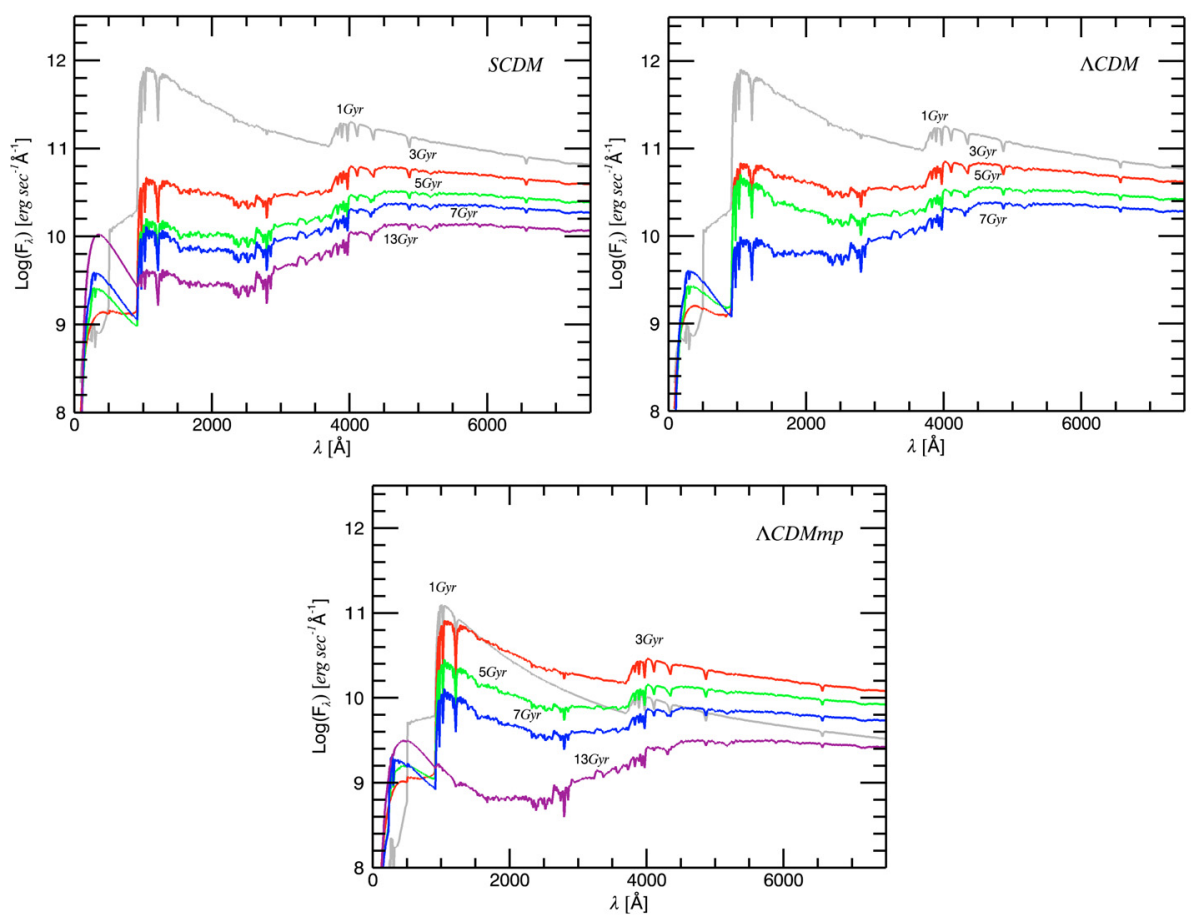

Fig. 4. SEDs of the model galaxies for the different cosmological scenarios, shown at different ages as indicated (SCDM upper left, $\Lambda \mathrm{CDM}$ upper right, $\Lambda \mathrm{CDM}_{\mathrm{mp}}$ lower panel). All curves refer to the rest-frame fluxes. Ages are in Gyr. corresponding SSP with same mass, age and chemical composition. During the evolution, the galaxy starts from an initial protogalaxy to end up to a final stage where the structure resembles that one of a spheroidal system. While the galaxy builds up itself, young star-particles have blue colors. Later, as the galaxy ages, the stellar component gets redder and redder.

Integrated magnitudes and colors. The evolution of the integrated magnitudes in the rest-frame, for the SCDM model galaxy, is shown in Figs. 6, for the VEGAmag in the BessellBrett photometric system (left panel) and ABmag in the SDSS (right panel), respectively.

The temporal evolution of magnitudes mirrors the history of star formation (see Fig. 1): in brief the magnitudes decrease as the galaxy gets the peak of SFR and hence becomes more luminous. Afterwards, the magnitudes increase following the SFR that declines rapidly. Figure 6 shows the magnitudes $M_{B}, M_{V}$, $M_{K}$, and $M_{1550}$ of the SCDM case for the Bessell-Brett system (left panel). The 1550-magnitude, that probes the UV region of the spectrum, weights the star formation at each epoch and reproduces the trend seen in the SFR. In the right panel of Fig. 6 we show the magnitudes in the SDSS photometric system of the same model. Although to lower extent, the same effect is visible in the $u$ and $g$ bands where the evolution is less smooth than for the other three bands.

The temporal evolution of colors is shown in the two panels of Fig. 7. Once again, the differences between models are primarily due to their SFH. In brief, the $\Lambda$ CDM and SCDM models have the same prescription for the SFR; therefore they have similar SFHs (both qualitatively and quantitatively) and similar color evolution. In the $\Lambda \mathrm{CDM}_{\mathrm{mp}}$ case with the multi-phase ISM, star formation is more gradual and lasts longer, the peak of activity is lowered and shifted to older ages as compared to the $\Lambda C D M$ case. The total mass assembled by the models is almost equal and the different behavior obtained with the two star formation prescriptions is likely due to the different time scales required to form new stars (see Merlin \& Chiosi 2007, for details).
To assess the quality of the integrated colors of our models we compare them to observed colors of a sample of nearby galaxies taken from the SDSS-DR7 database. We select the sample with the following criteria: the galaxies must have redshift $z \lesssim 0.01$, the galaxy images should be taken far away from the CCD edges, they should unsaturated, and finally, the photometric errors in each bands should be smaller than 0.1 mag (good signal to noise ratios). With these criteria we obtain a sample of 5986 galaxies. Figure 8 shows the galaxies in a color-color diagram and compare them with the rest-frame color evolution of the models. The age increases as indicated by the arrow. Galaxies classified as ETGs, using the exponential $\left(P_{\exp }\right)$ and de Vaucouleurs' $\left(P_{\mathrm{deV}}\right)$ profile likelihoods (see Shimasaku et al. 2001; Strateva et al. 2001, for more details on the SDSS morphological classification), are indicated with empty circles, whereas late type galaxies (LTGs) are shown with black dots. The sample has been corrected for the extinction. The color evolution of our simulations is indicated with filled squares, empty circles, and filled triangles for the three models. Remarkably, simulated colors and data nicely agree, in particular for ages older than 5 Gyr.

\subsection{Distribution of the stellar populations in the color-magnitude diagram}

It might be worth of interest to explore the age-metallicity range spanned by the stellar populations of a galaxy by looking at their distribution in the color-magnitude diagram (CMD), in analogy to what currently made for stars in clusters and fields.

The CMDs of Fig. 9 show SSPs of different age and metallicity, and the star-particles of the galaxy simulations (the filled circles). To calculate the magnitude of the SSPs we have assigned them the same mass of the star-particles in the NB-TSPH simulations. The solid lines show the evolutionary path followed by SSPs of different metal content as their integrated luminosity and color change as a function of the age. Along each line the age goes from 0.1 Gyr to 14 Gyr. This is the analog of the evolutionary path followed by stars of given mass and chemical 

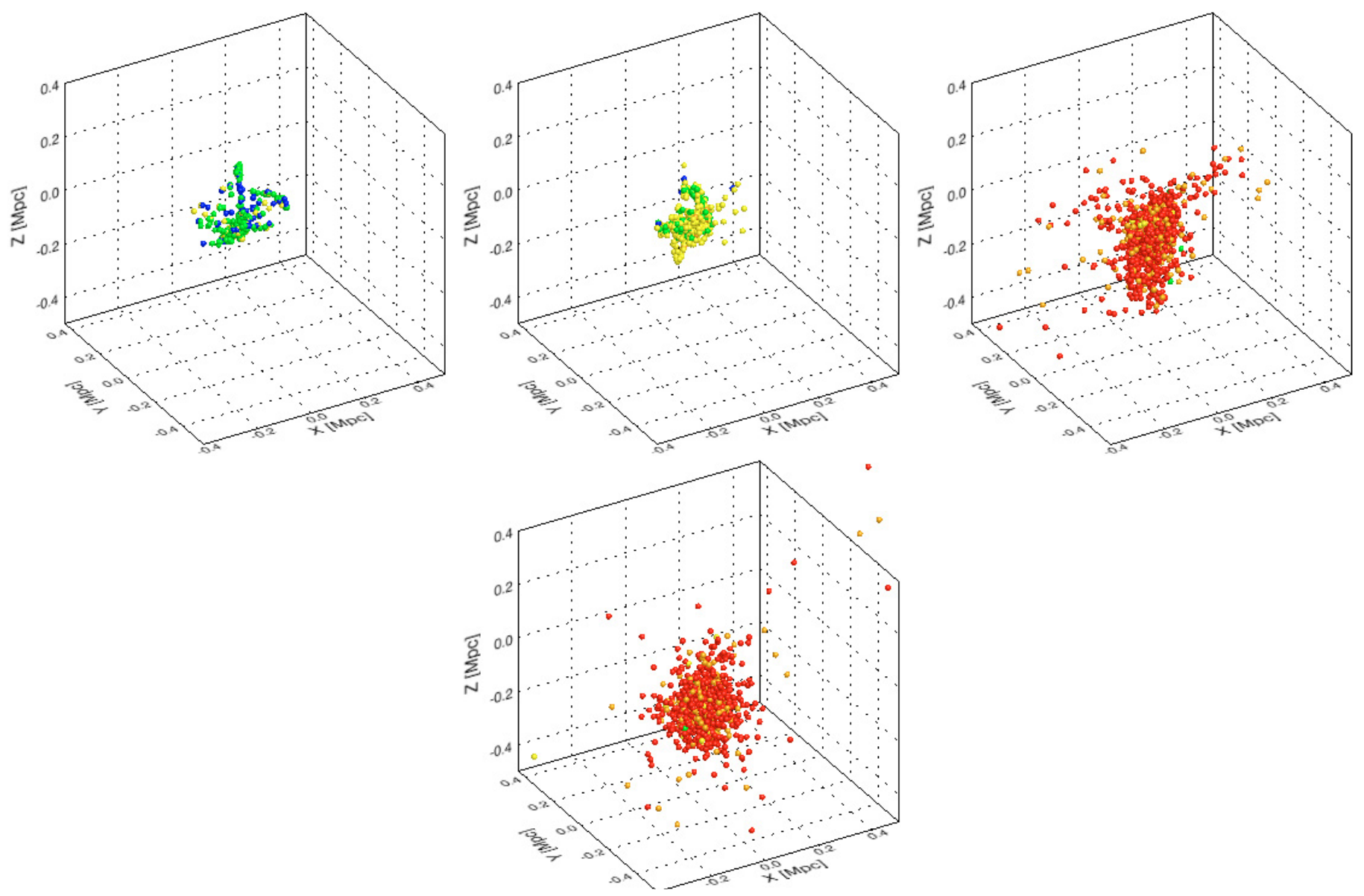

\section{$\mathrm{B}-\mathrm{V}<0.1 \cdot 0.1<\mathrm{B}-\mathrm{V}<0.3 \quad 0.3<\mathrm{B}-\mathrm{V}<0.5 \quad 0.5<\mathrm{B}-\mathrm{V}<0.6 \quad \mathrm{~B}-\mathrm{V}>0.8$}

Fig. 5. Three-dimensional view of the SCDM simulation for four epochs (1 Gyr upper left panel, 2 Gyr upper right, 5.5 Gyr lower left, and 13 Gyr lower right). The color code of each star-particle corresponds to the value of the $(B-V)$ color as indicated.

composition. The dashed lines show SSPs of the same age (as indicated) and different metallicity. Along each line the metallicity goes from $Z=0.0001$ to $Z=0.07$. The CMDs allow us to catch immediately how the stellar populations of a model galaxy (as represented by its star-particles) distribute in age and metallicity. The analog of this situation for real stars would be a CMD built up with the integrated magnitudes and colors of the stellar clusters of a galaxy, for instance the clusters of the LMC and $\mathrm{SMC}$, the only difference is that while real clusters have different mass our star-particles are all with the same mass. However, this is a point of minor relevance here.

In the $(V-K)$ vs. $V$ diagram we can see how star-particles distribute at varying the metallicity: in the SCDM galaxy at the age of 13 Gyr the vast majority of star-particles are very old. The bulk of stars distribute along the lines of very old ages and span all the values of metallicity. This means that the $(V-K)$ color tests the metallicities differences, more than the age. However there is a fraction of younger stars that tends to crowd the region comprised between $Z=0.019\left(Z_{\odot}\right)$ and $Z=0.070\left(3.5 Z_{\odot}\right)$.

In the $(1550-V)$ vs. $V$ diagram, on the other hand, the bulk of the stars distribute along the $Z=0.019$ line and have ages going from very old to very young. Since the metallicity has a lower effect, this diagram can be used to infer the gross age of the stellar content of a galaxy.

To get a deeper insight of the whole problem, in Figs. 10, from left to right, we show the distribution of the stellar populations in the $g-(g-i)$ plane for the SCDM model at the age of 13 Gyr. In each panel, stars younger than a certain limit (that varies from panel to panel as indicated) are plotted as light dots, the remaining ones as dark dots. The insert in the upper right corner in each panel shows the position on the $x y$ projection plane of such stars (the light and dark dots). As expected, owing to the residual star formation activity (say after the first $5 \mathrm{Gyr}$ ) stars of younger and younger age tend to concentrate toward the galactic center. Keeping the same color-code, in Fig. 11, we show how the stars of different age distribute in metallicity. As expected the stars of very low metallicity are in general very old, whereas at increasing metallicity stars of any age are possible. Finally, in Fig. 12 we show how stars of different age and metallicity spatially distribute within the galactic volume.

In order to validate the quality of our photometry, we compare the colors of our SSPs and star-particles of the NB-TSPH simulation with the colors of observed real stellar clusters. This is shown in Fig. 13, where we display: the integrated $(U-B)_{0}$ and $(B-V)_{0}$ colors of the LMC clusters by Bica et al. (1991) (open rhombs); the Galactic Globular Clusters by Harris (1996) limited to a few indicative cases (asterisks); the SSPs with different metallicity indicated by the dotted dashed lines (the heavy solid line is for $Z=0.008$, the typical mean metallicity of the LMC); finally the star-particles of the SCDM galaxy model (small open circles). Data and theoretical predictions seem to agree each other but for the youngest clusters of the LMC which tend to 

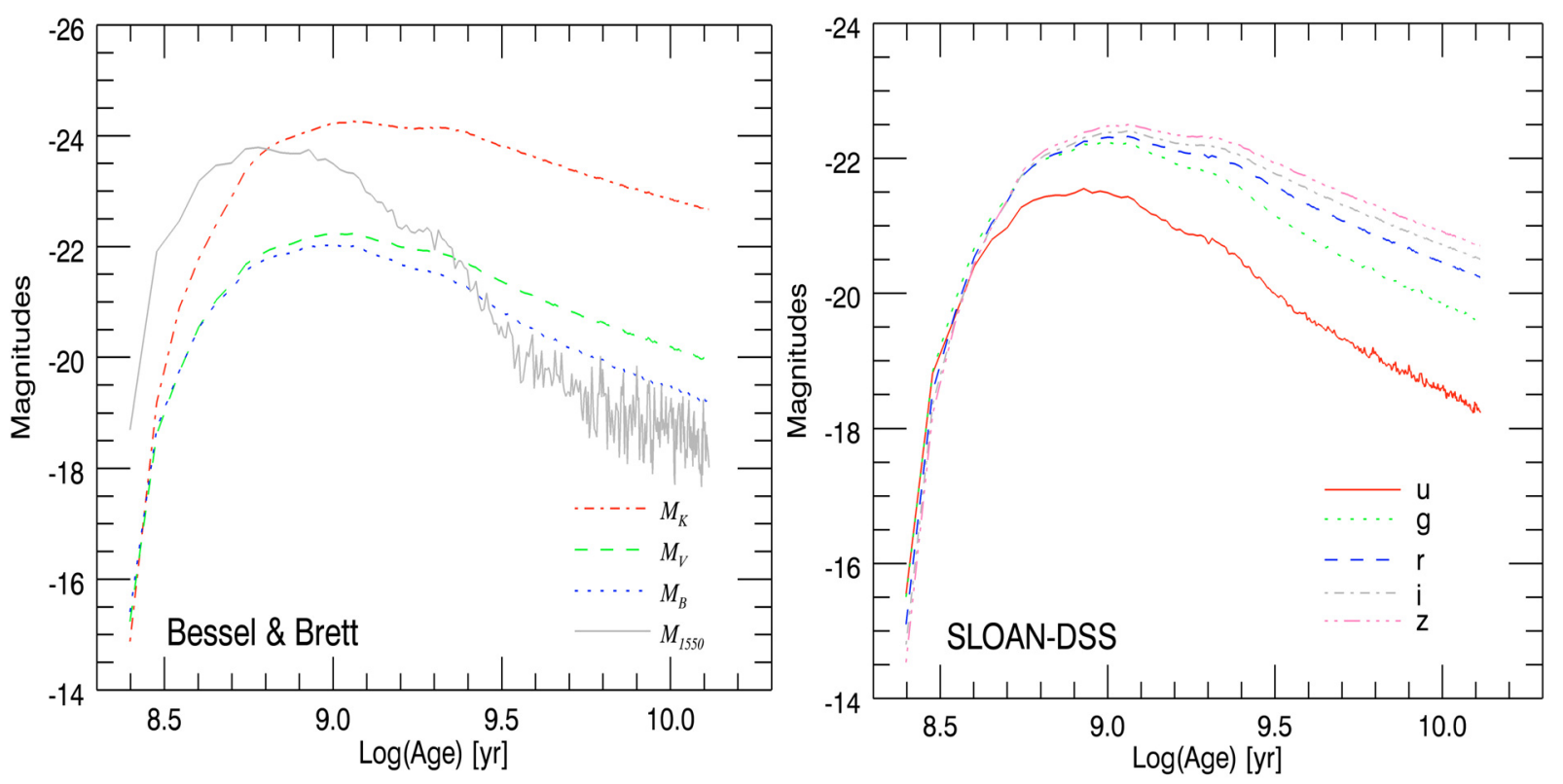

Fig. 6. Left panel: rest-frame evolution of the total absolute Bessell \& Brett magnitudes, $M_{K}, M_{V}, M_{B}$, and $M_{1550}$, of the SCDM model. Right panel: the same as in the left panel but for the $u, g, r, i$, and $z$ passbands of the SDSS photometric system.
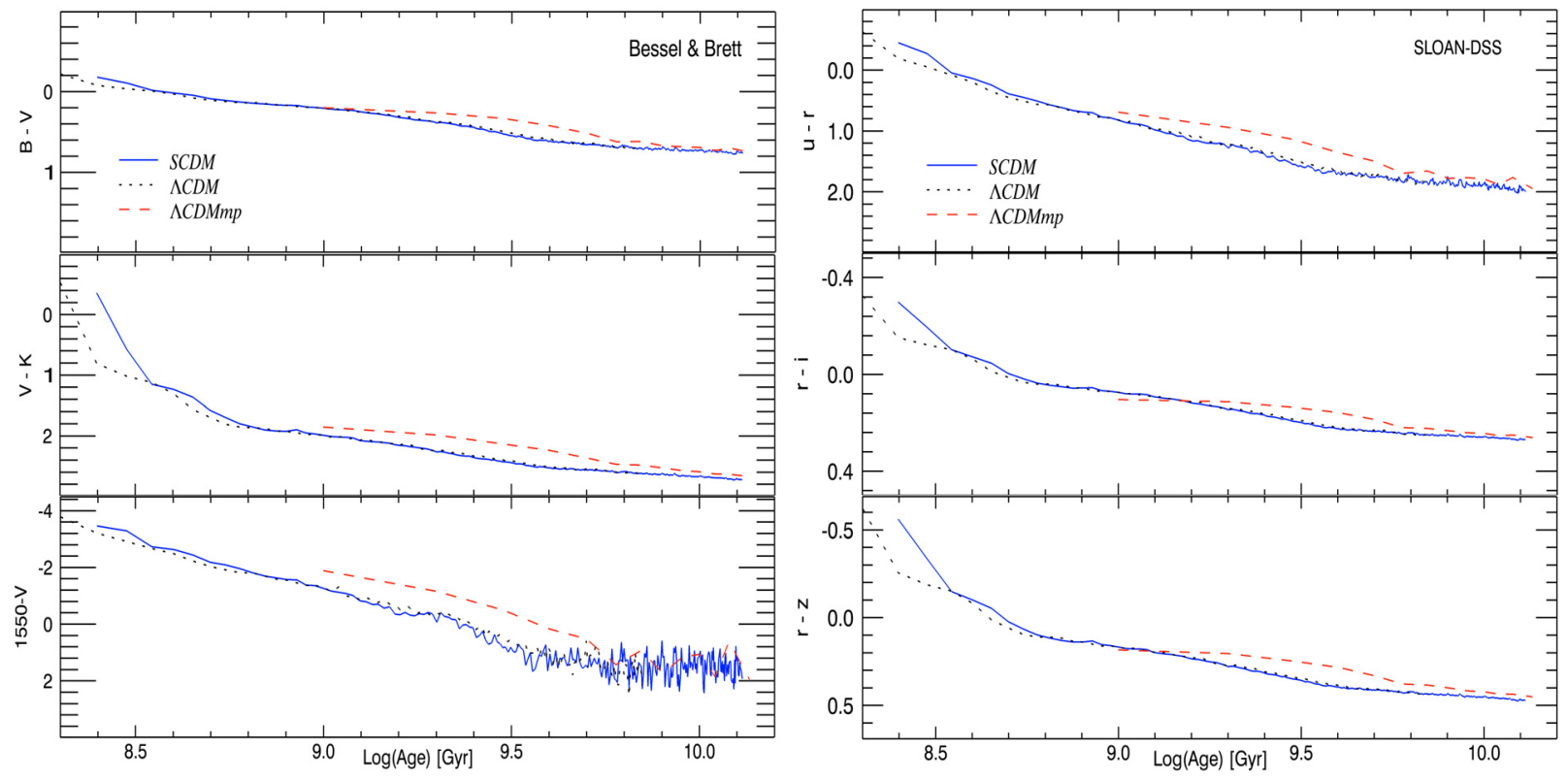

Fig. 7. Left panel: rest-frame evolution of the $B-V, V-K$, and $1550-V$ colors for the Bessell-Brett photometric system shown by our model galaxies as indicated. Right panel: the same as in the left panel but for SDSS colors $u-r, r-i$, and $r-z$. In both panels the $\Lambda$ CDM $\mathrm{mp}_{\mathrm{mp}}$ case is plotted only for age older than $1 \mathrm{Gyr}$ for the sake of clarity. For younger ages it runs very close to the other two cases.

scatter above the line for $Z=0.008$. However, this is less of a problem as a plausible explanation has been advanced by Girardi et al. (1995). Therefore, the above agreement between theory and data secures that our photometry is carefully calculated.

\section{Cosmological spectro-photometric evolution: theory and data}

The advent of the modern giant telescopes has opened a new era in observational cosmology and galaxy evolution can be traced back to very early stages. In this context, deep multi-color imaging surveys provide a powerful tool to access the population of faint galaxies with relatively high efficiency. These surveys span the whole spectral range from the UV to the near-IR bands, enabling galaxy evolution to be followed on a wide range of redshifts. Therefore it is worth looking at the cosmological evolution of our model galaxies and compare it with modern data. Since galaxies are observed at different redshifts in an expanding Universe, we need the so-called $K$-correction and $E$-corrections that can be easily derived together with magnitudes and colors from the population synthesis technique (see Guiderdoni \& Rocca-Volmerange 1987; Rocca-Volmerange \& Guiderdoni 1988; Guiderdoni \& Rocca-Volmerange 1988; Bressan et al. 1994). Finally, we compared the colors of our models with those of two sample of ETGs extracted from the COSMOS and GOODS databases. 
R. Tantalo et al.: Spectro-photometric models of early type galaxies

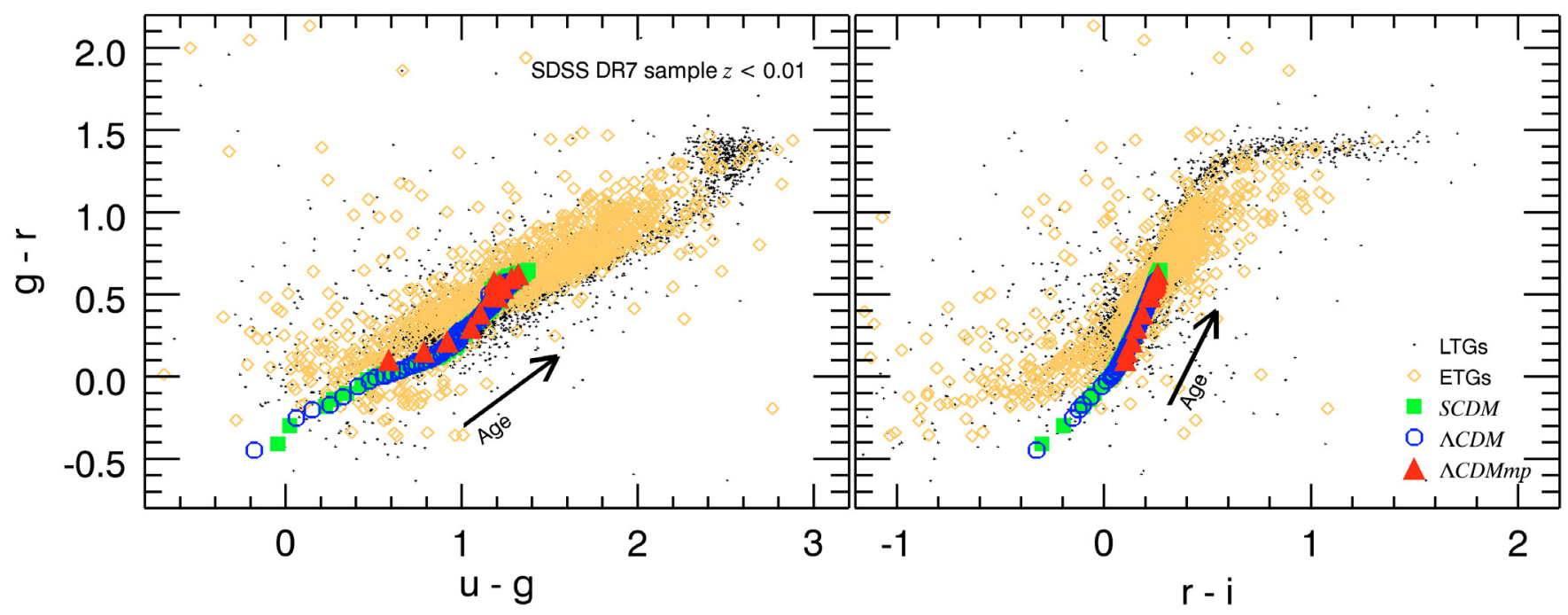

Fig. 8. Validation of the integrated colors of our models compared to the observational data for a sample of nearby galaxies. We show the color-color distribution of a sample of galaxies from DR7 SDSS. The data are selected for $z \leq 0.01$. Small dots and diamonds are for late type galaxies (LTGs) and ETGs, respectively. The evolution of SCDM (filled squares), $\Lambda$ CDM (empty circle), and $\Lambda$ CDM ${ }_{\mathrm{mp}}$ (filled triangles) in the rest-frame are overlapped with age increasing as indicated.
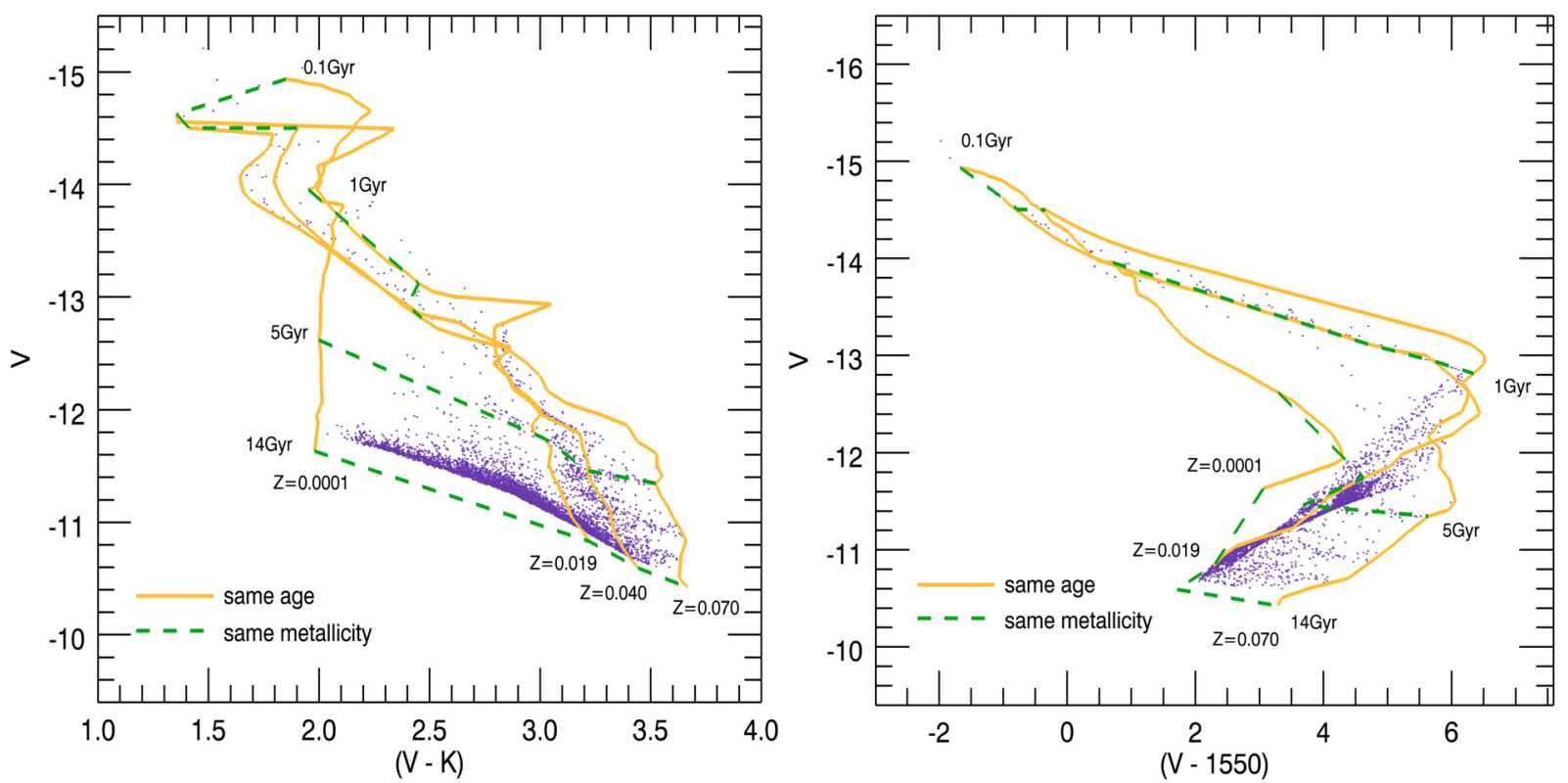

Fig. 9. Left panel: distribution of the stellar populations in the $(V-K)-V$ plane for the SCDM model at the age of $T=13$ Gyr. The SSPs color grid is overlapped for interpretation of ages and metallicities. Right panel: the same as in the left panel but for the $(1550-V)-V$ plane. See the text for details.

\subsection{Evolutionary and cosmological corrections}

When we consider a source observed at redshift $z$, we need to remember that a photon observed at a wavelength $\lambda_{\mathrm{o}}$ has been emitted at wavelength $\lambda_{\mathrm{e}}$. The two wavelengths are related by

$\lambda_{\mathrm{e}}=\lambda_{\mathrm{o}} /(1+z)$

A source with apparent magnitude $m$ measured in a photometric passband, is related to the absolute magnitude $M$, in the emission-frame passband, through the cosmological correction, $K_{\text {corr }}$, in the following way (Hubble 1936):

$m=M+D M+K_{\text {corr }}$, where $D M$ is the distance modulus, defined by

$D M=5 \log _{10}\left[\frac{D_{L}(z)}{10 \mathrm{pc}}\right]$,

being $D_{L}(z)$ the luminosity distance.

The above luminosity distance has been calculated with the same cosmology of the simulated galaxies $\left(\Omega_{\mathrm{M}}, \Omega_{\Lambda}, h_{0}\right)$. In particular, we have adopted the following equation (see Weinberg 1972; Hogg 1999; Kolb \& Turner 2000, for all details):

$D_{L}(z)=\frac{c}{H_{0}}(1+z) \int_{0}^{z} \frac{\mathrm{d} z}{\left[\Omega_{\mathrm{M}}(1+z)^{3}+\Omega_{\Lambda}\right]^{\frac{1}{2}}}$. 

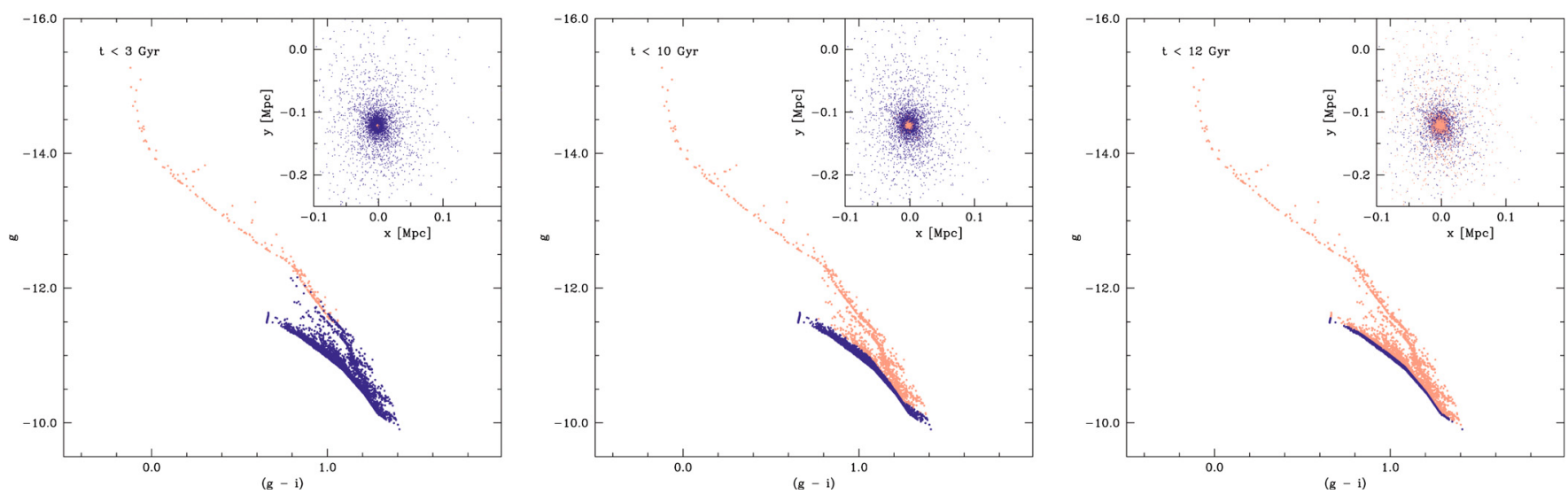

Fig. 10. Distribution of the stellar populations in the $(g-i)$ vs. $g$ plane for the SCDM model at the age of $T=13$ Gyr. Stars younger than a certain limit (that varies from panel to panel as indicated) are plotted as light dots. The insert in the upper right corner in each panel shows the position of such stars (with light colors) and of the remaining stars (dark dots). As expected, the residual star formation (say after the first 5 Gyr) tends to occur preferentially in the very central regions of the galaxy.
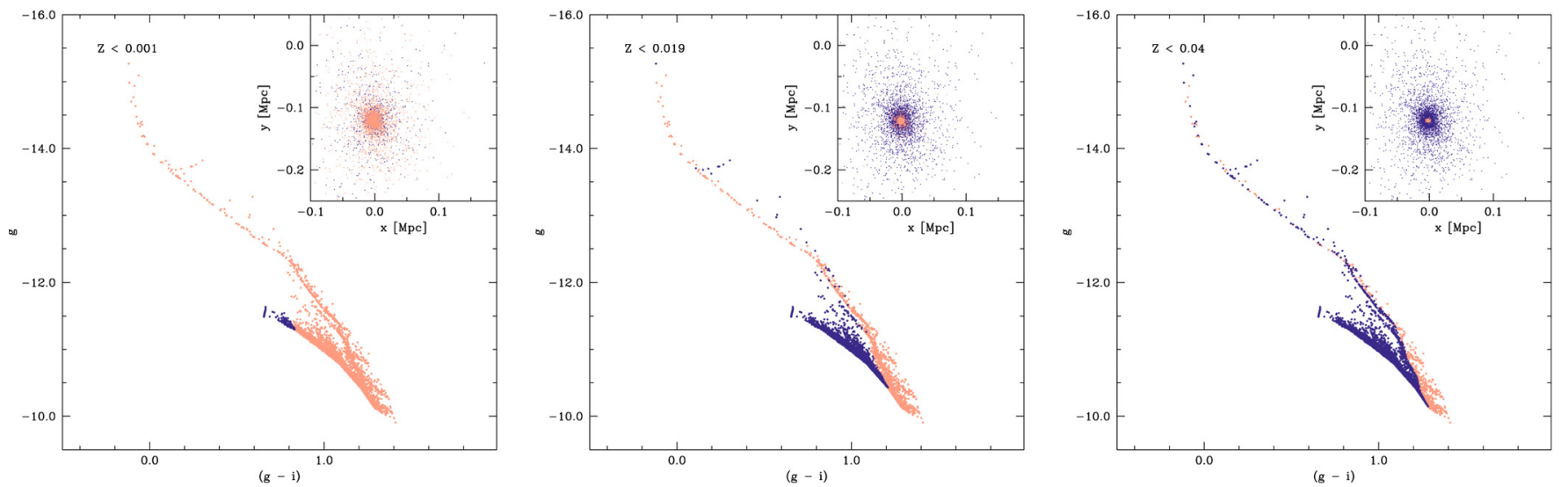

Fig. 11. Same as Fig. 10 but for stellar populations with different metallicity. As expected the stars of very low metallicity are in general very old, whereas at increasing metallicity stars of any age are possible.
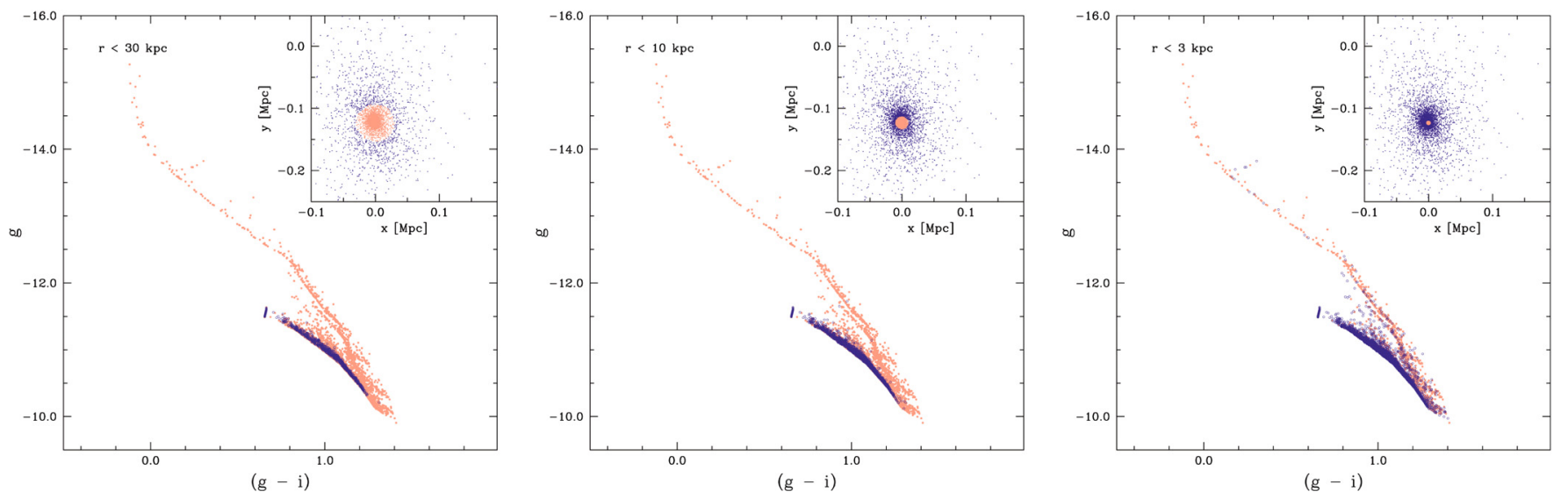

Fig. 12. Same as Fig. 10 but for stars of any age and metallicity but different locations in the galaxy.

If the source is at redshift $z$, then its luminosity is related to its spectral density flux (energy per unit time, unit area, and unit wavelength) by

$L\left(\lambda_{\mathrm{e}}\right)=4 \pi(1+z) D_{L}^{2} f\left(\lambda_{0}\right)$,

where $f\left(\lambda_{0}\right)$ is the monochromatic flux of a galaxy that has been computed as defined in Eq. (4).
Finally, the $K_{\text {corr }}$ in Eq. (6) is

$K_{\text {corr }}=2.5 \log _{10}(1+z)+2.5 \log _{10}\left[\frac{L\left(\lambda_{0}\right)}{L\left(\lambda_{\mathrm{e}}\right)}\right]$

(see the definition by Oke \& Sandage 1968). This means that to make a fair comparison between objects at different redshifts, we must derive the rest-frame photometric properties of 
R. Tantalo et al.: Spectro-photometric models of early type galaxies

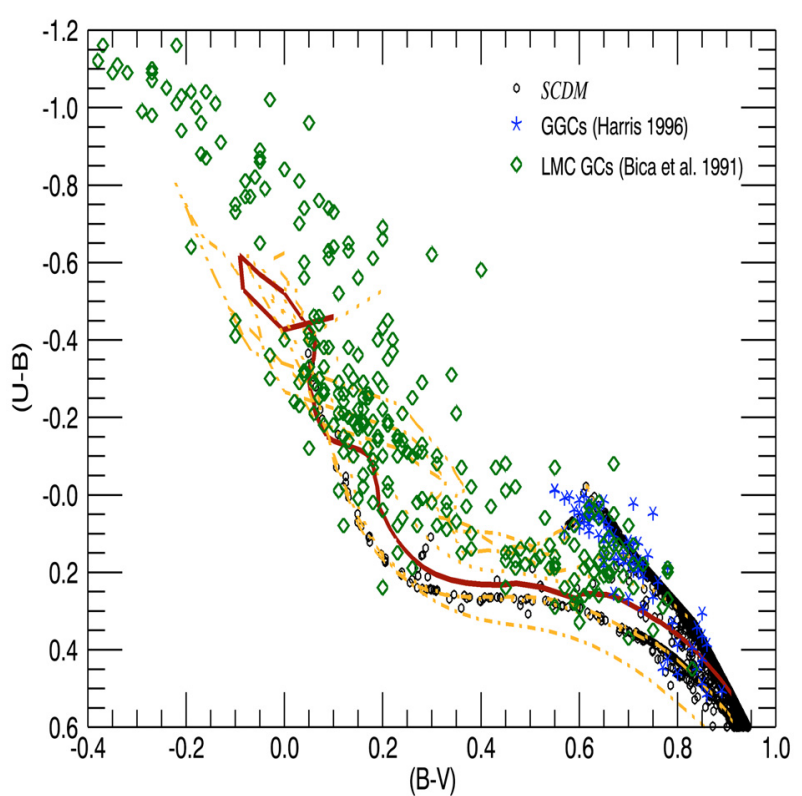

Fig. 13. Integrated $(U-B)_{0}$ and $(B-V)_{0}$ colors of the LMC clusters by Bica et al. (1991), open rhombs; the galactic globular clusters by Harris (1996), asterisks; the SSPs with different metallicity, dotted-dashed lines, the heavy solid line is the one with the metallicity typical of the LMC, i.e. $Z=0.008$ ); finally, the star-particles of the SCDM galaxy model, the small open circles. Data and theoretical predictions seem to agree each other.

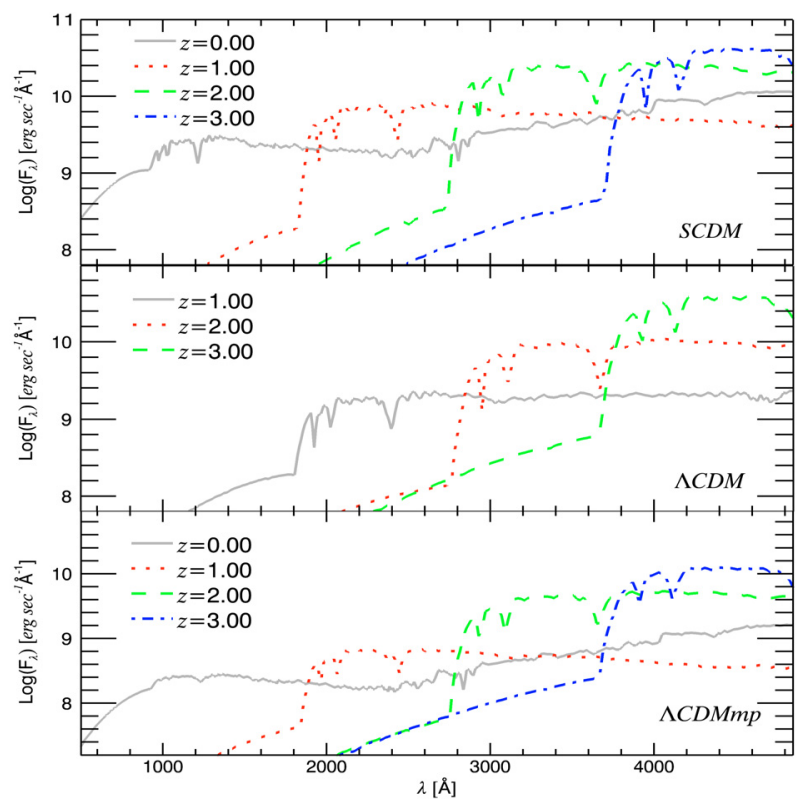

Fig. 14. Red-shifted spectra at different redshifts for the three galaxy models. Internal extinction is taken into account.

our observed galaxies (magnitudes, colors, etc.) by applying $K$-corrections.

In addition, we must also correct these rest-frame quantities for the expected evolutionary changes over the redshift range studied, by applying the so called evolutionary corrections, $E_{\text {corr }}$. The $E_{\text {corr }}$ are usually derived assuming a model for the galaxy SED and calculating its evolution with the redshift. In this way we can recover the evolution of the absolute magnitudes and colors as a function of the redshift $z$, including the effect of the $K$ and $E$-corrections on the SED of our models.
Following Guiderdoni \& Rocca-Volmerange (1987), the cosmological $K(z)$ and evolutionary $E(z)$ corrections are conventionally given in terms of magnitude differences:

$K(z)=M\left(z, t_{0}\right)-M\left(0, t_{0}\right)$,

$E(z)=M\left(z, t_{z}\right)-M\left(z, t_{0}\right)$,

where $M\left(0, t_{0}\right)$ is the absolute magnitude in a passband derived from the rest frame spectrum of the galaxy at the current time, $M\left(z, t_{0}\right)$ is the absolute magnitude derived from the spectrum of the galaxy at the current time but red-shifted at $z$, and $M\left(z, t_{z}\right)$ is the absolute magnitude obtained from the spectrum of the galaxy at time $t_{z}$ and red-shifted at $z$.

From Eq. (6) the apparent magnitude, in some broad-band filter and at redshift $z$, is given by:

$m(z)=M(z)+E(z)+K(z)+D M(z)$.

Obviously, the relation $t=t(z)$, between the cosmic time $t$ and the redshift $z$ of a stellar population formed at a given redshift $z_{f}$, depends on the cosmology considered and the parameters adopted. Following Kolb \& Turner (2000):

$$
t(z)=\frac{1}{H_{0}} \int_{z}^{\infty} \frac{\mathrm{d} z}{(1+z)\left[\Omega_{\mathrm{M}}(1+z)^{3}+\Omega_{\Lambda}\right]^{\frac{1}{2}}}
$$

\subsection{Extinction}

Before calculating the $E_{\text {corr }}$, it is worth applying to the theoretical SEDs the effect of extinction of the stellar luminosity caused by the presence of a certain amount of metal-rich gas so that the SEDs get closer to the real ones. Although the task is a complicate issue requiring a careful analysis (Piovan et al. 2003, 2006a,b), for the purposes of the present study, the effect of extinction can be evaluated using the relation proposed long ago by Guiderdoni \& Rocca-Volmerange (1987):

$\tau_{\lambda}=3.25\left(1-\omega_{\lambda}\right)^{0.5}\left(A_{\lambda} / A_{V}\right)_{\odot}\left[Z(t) / Z_{\odot}\right]^{1.35} G(t)$,

where $\tau_{\lambda}$, the effective optical thickness of the gaseous component at a given $\lambda$, is a function of: (i) the albedo $\omega_{\lambda}$ of the grains for which the mean values of 0.4 taken from Draine \& Lee (1984) has been used; (ii) the extinction law $A_{\lambda} / A_{V}$ (Cardelli et al. 1989); (iii) and finally the metallicity $Z(t)$ and gas fraction $G(t)$.

The monochromatic flux of the galaxy with the inclusion of the effect due to extinction, $F_{\lambda \text {,ext }}$, can be expressed in function of the monochromatic flux of the rest-frame SED of the model galaxy $F_{\lambda}$ (see Eq. (4)):

$F_{\lambda, \text { ext }}=F_{\lambda} \frac{1-\exp \left(-\tau_{\lambda} \sec i\right)}{\tau_{\lambda} \sec i}$,

where the right-hand part of the expression takes into account the transmission function for an angle of inclination $i$; we adopt here $i=45$. Although this relation was originally derived for disk galaxies, it can be safely used also in our case.

The effect of extinction is included in our SEDs using $Z(t)$ and $G(t)$ obtained from the NB-TSPH simulations. Internal extinction may significantly redden the colors, the effect being particularly important on the color-redshift relation. To illustrate the point, in Fig. 15 we compare the SEDs of the SCDM and $\Lambda \mathrm{CDM}_{\mathrm{mp}}$ models at the age of $13 \mathrm{Gyr}$ with and without extinction, as indicated. First of all the two SEDs are different even 


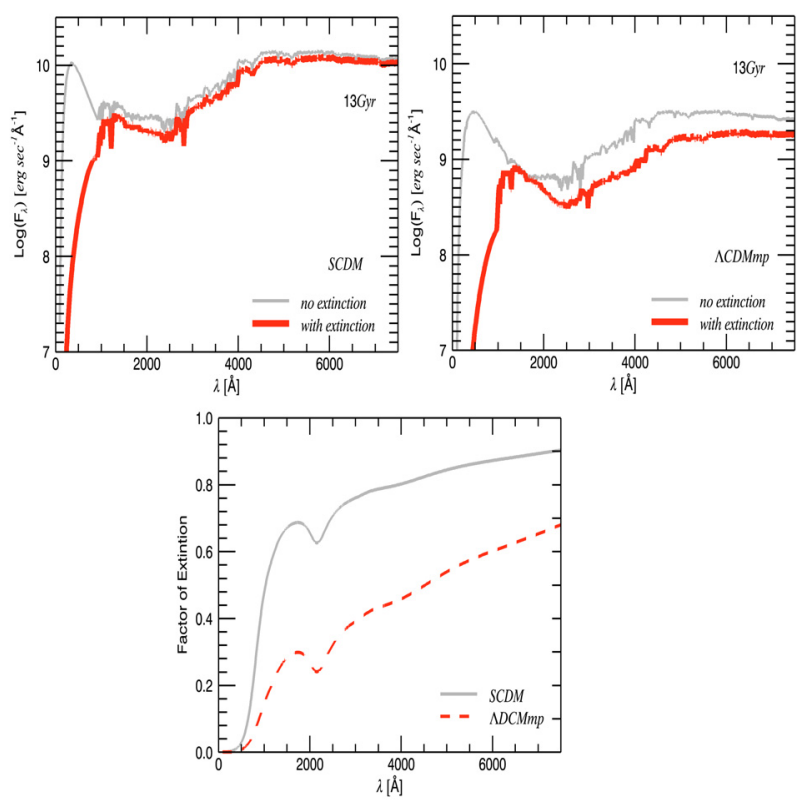

Fig. 15. Top panels: comparison between the SEDs of the SCDM and $\Lambda \mathrm{CDM}_{\text {mp }}$ models at the age of $13 \mathrm{Gyr}$ with extinction (thick line) and without extinction (thin line). Bottom panel: the extinction factor for the two models as indicated.

neglecting extinction (they reach indeed different levels of flux), see the top panels of Fig. 15. This simply reflects the final lower mass in stars of the $\Lambda \mathrm{CDM}_{\mathrm{mp}}$ model with respect to SCDM (a factor of three lower). Second, the effect of extinction is different in the two models (top panels of Fig. 15). This simply reflects the different metallicity and gas content at the age of 13 Gyr. These are $Z(t)=0.0214$ and $G(t)=0.257$ in SCDM and $Z(t)=0.0513$ and $G(t)=0.441$ in $\Lambda \mathrm{CDM}_{\mathrm{mp}}$. The factor $\left[1-\exp \left(-\tau_{\lambda} \sec i\right) / \tau_{\lambda} \sec i\right]$ of Eq. (16) with $i=45^{\circ}$ is shown in the bottom panel of Fig. 15.

There is a final point to consider, i.e. the intrinsic reliability of the magnitudes and colors derived from SEDs as a function of the redshift. To illustrate the point, in Fig. 14 we display the redshifted spectrum with extinction of the models for some values of $z$. The spectra show a drastic change in the slope that occurs at a certain wavelength whose value increases with the redshift. This effect is because the rest-frame theoretical spectra have a lower limit of $912 \AA$ and that the extension to $\lambda<912 \AA$ has been made by simply assuming black-body spectra. The real spectrum short-ward of $912 \AA$ could be different from a pure black-body. The effect of this approximation should be taken into account in the computation of the colors in any photometric system. In other words, magnitudes and colors that contain flux originated in the $\lambda<912 \AA$ interval become more and more uncertain at increasing redshift. This is illustrated in Fig. 16 which displays the quantity $\lambda_{\mathrm{obs}}=\lambda_{e m}(1+z)$. The shaded area shows the region of intrinsic uncertainty due to the above effect. It emerges from this that at redshift of $z=3$ (observational data in the surveys considered reach this redshift) magnitudes are considered accurate at wavelengths $\lambda>4100 \AA$.

\subsection{Comparison with data}

The advent of large-scale space and ground-based surveys in a wide range of wavelengths is giving us unprecedented access to statistically large populations of galaxies at different redshifts (and also environments). The practical use of these immense

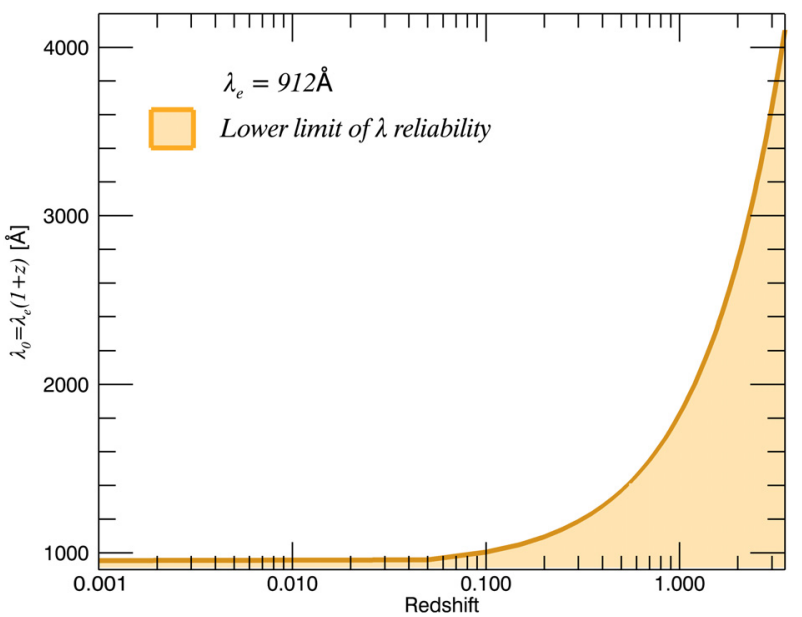

Fig. 16. Reliability of magnitudes and colors as function of the redshift is because the theoretical spectra in the population synthesis algorithm do not extend at wavelengths shorter than $\lambda=912 \AA$ and replaced by black-body spectra (approximation).

databases requires some caution as far as galaxy detections, redshift assignment, galaxy classification, and galaxy selection are concerned. Prior to anything else it is worth recalling that owing to the enormous amounts of data to handle, the data acquisition process is usually made following automatic procedures that deserve some remarks.

Detection. At $z>5$, traditional optical bands, e.g. $U B V R$, fall below the rest-frame wavelength that corresponds to the Lymanbreak spectral feature $(1216 \AA$ ), where most of the stellar radiation is extinguished by interstellar or intergalactic hydrogen. Because of this, galaxies at $z>5$ are practically invisible at those photometric bands, and even if they were detected, their colors would provide very little information about their stellar population. The color selection technique, e.g. the UGR selection of Lyman-Break Galaxy (LBGs) by Steidel et al. (1996, 1999) and Steidel et al. (2003), has been used in some surveys to identify galaxies at high redshift, dramatically improving the efficiency of spectroscopic surveys at $z>3$.

Redshift assignment. Photometric redshifts are the logical extension of color selection by estimating redshifts and SEDs from many photometric bands. Unlike color selection, photometric redshifts take advantage of all available information, enabling redshift estimates along with the age, star formation rate and mass.

Morphological classification. One of the main characteristics of deep photometric surveys is the richness of detected objects, where a significant fraction of them appear as point sources that cannot be neither easily distinguished from real stars nor morphologically classified. Therefore, the classification by means of morphological and photometric criteria is a crucial issue. In this paper, we consider two deep catalogues that allow us to select good samples of ETGs.

Selection. Morphological selection of ETGs can be made using automated pipelines that isolate objects on the basis of their twodimensional light distributions: this is the case of the COSMOS 
Table 4. COSMOS survey: telescopes and optical/IR bands.

\begin{tabular}{llc}
\hline \hline Telescope & \multicolumn{1}{c}{ Filters } & Band width (in $\AA$ ) \\
\hline CFHT & $u^{*}, i^{*}$ & $3200-1000$ \\
CTIO & $K \mathrm{~s}$ & $9000-25000$ \\
HST-ACS & $F 814 W(i$-band) & $4000-11000$ \\
KPNO & $K \mathrm{~s}$ & $9000-25000$ \\
SDSS & $u, g, r, i, z$ & $3200-11000$ \\
Subaru & $B_{J}, V_{J}, g^{+}, r^{+}, i^{+}, z^{+}, \mathrm{NB} 816(i$-band $)$ & $4000-11000$ \\
\hline
\end{tabular}

survey (see below). On the other hand, in the case of GOODS, it is possible to select more accurately these objects by correlating a catalogue of photometric and spectroscopic redshifts with a morphological one.

\subsection{COSMOS survey}

In this analysis, we use the Cosmic Evolution Survey COSMOS official photometric redshift catalogue (Scoville et al. 2007), designed to probe the evolution of galaxies in the context of their large scale structure out to moderate redshift. Details of the COSMOS catalogue are described in Capak et al. (2007) and Mobasher et al. (2007). It covers a 2 square degree area with deep panchromatic data and includes objects whose total $i$ magnitudes $\left(i^{+}\right.$or $\left.i^{*}\right)$ are brighter than 25 .

The COSMOS multi-band catalogue embraces data from different telescopes, as listed in Table 4, and presents imaging data and photometry that cover various photometric bands between $0.3 \mu \mathrm{m}$ and $2.4 \mu \mathrm{m}$. The catalogue was generated using SExtractor (Bertin \& Arnouts 1996) and contains photometry measured over 3 arcsec diameter apertures for all the bands. All magnitudes are in the $\mathrm{AB}$ system. The cosmology adopted is: $H_{0}=70 \mathrm{~km} \mathrm{~s}^{-1} \mathrm{Mpc}^{-1}, \Omega_{\mathrm{M}}=0.3$ and $\Omega_{\Lambda}=0.7$. It also contains photometric redshifts, 68 and 95 percent confidence intervals, and spectral types calculated with two different packages: the Mobasher et al. (2007) and the Bayesian Photometric Redshift (BPZ by Benítez 2000).

Such a large database needs an automated and objective morphological classification procedure separating ETGs from other objects. The goal is achieved using the parameter $T_{\text {phot }}$, which is based on the spectral type. Objects with $T_{\text {phot }} \leq 1.5$ correspond to ETGs, those with $T_{\text {phot }}>1.5$ to all the remaining types.

For our model galaxies we generate magnitudes and colors in the same photometric system of COSMOS, and calculate the cosmological and evolutionary corrections suited to the cosmological background in use. Colors and their cosmological evolution in different bands are shown in Figs. 17-19, where the $B_{J}-r^{+}$(Subaru), $r^{+}-K$ s (Subaru/KPNO), and the $g-r$ (SDSS) colors are plotted as an example. It is worth calling attention that the model $\Lambda C D M$ is plotted from $z_{\text {ini }}$ down to $z \sim 1$ because it has been stopped at the age of 7 Gyr. For the sake of comparison, first we display all the galaxies from the survey, independently of their classification (light stars) and, superposed to them, we mark in dark stars the sub-sample of ETGs selected following the classification suggested by the automated pipeline $\left(T_{\text {phot }} \leq 1.5\right)$. The photometric evolution for our three model galaxies is also shown: the solid line is for the SCDM, the dotteddashed line is for the $\Lambda \mathrm{CDM}$ model, and the dotted line is for the $\Lambda \mathrm{CDM}_{\mathrm{mp}}$ case.

The models follow the general trend of the observations and, in particular, are marginally consistent with the group of ellipticals up to $z \sim 1$ beyond which the data are too poor to say anything. The observed ellipticals are indeed redder than the mean

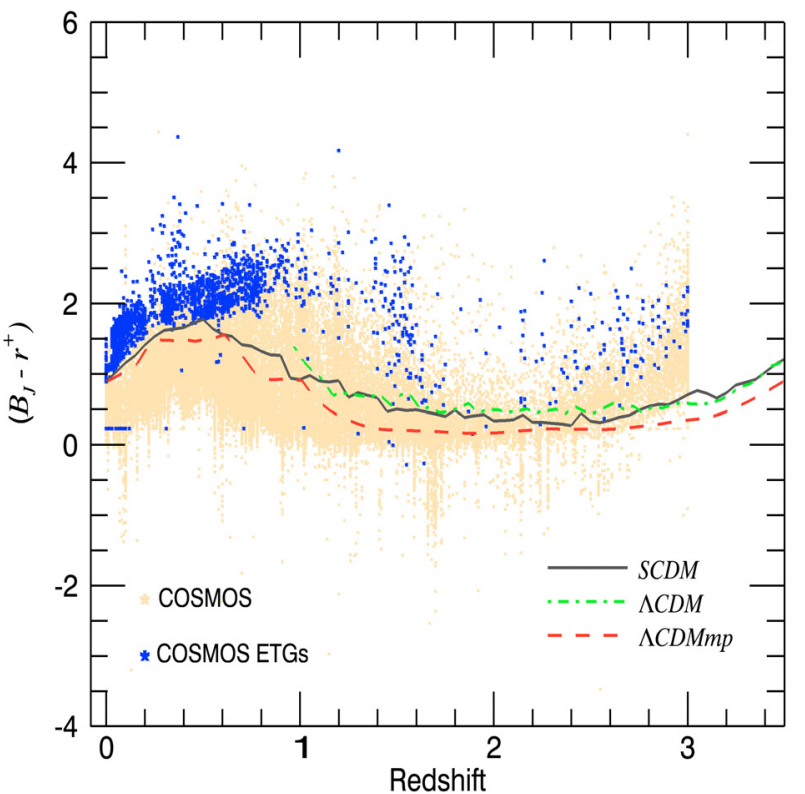

Fig. 17. Cosmological evolution with redshift for the $\left(B_{J}-r^{+}\right)$color of the COSMOS survey. Both passbands are those of the Subaru Telescope. All galaxies of the catalog are shown in light stars. The ETGs selected with the pipeline morphological $T_{\text {phot }}<1.1$ parameter are marked in dark stars. The galaxy models for the three different cosmological scenarios are shown superimposed to the data, continuous and dotted lines as labelled. The $\Lambda$ CDM case is shown for $z>1$.

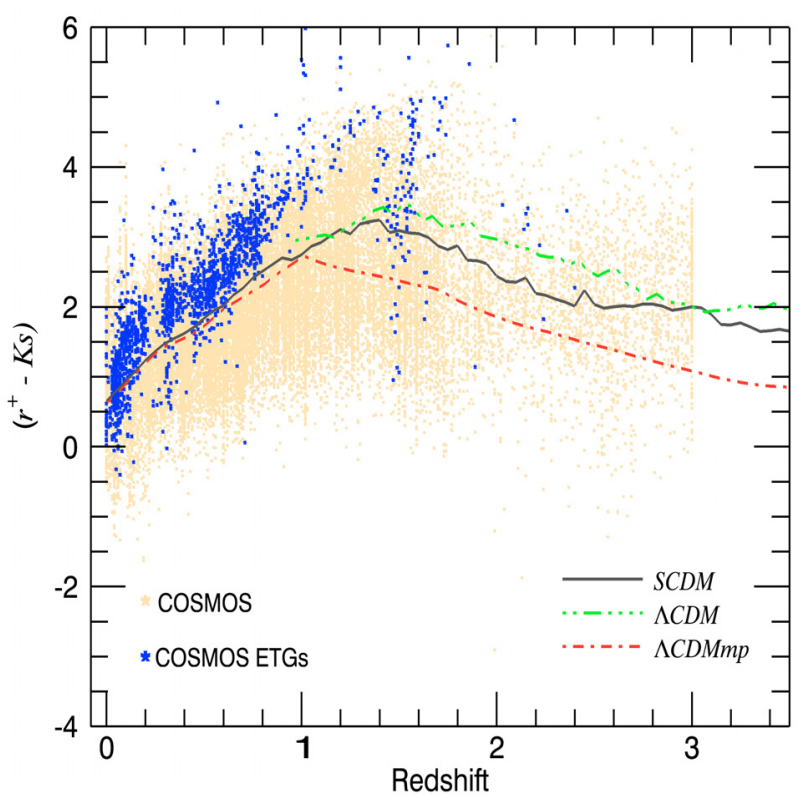

Fig. 18. Same as Fig. 17 but for the $\left(r^{+}-K \mathrm{~s}\right)$ colors $\left(r^{+}\right.$band from Subaru and $K$ s band from KPNO).

value of the data and theoretical predictions. Concerning the theoretical values, their bluer colors can be ascribed to the tail of star formation extending to the present. Although this minor stellar activity does not significantly affect the gross features of the models (structure, mass distributions etc.) it certainly affect the colors making them bluer than desired and expected. This secondary star formation activity is likely a spurious effect (work is progress to cope with this). Another point of uncertainty could reside in the selection criteria to identify ETGs. 
Table 5. GOODS database: telescopes and optical/IR bands.

\begin{tabular}{llc}
\hline \hline \multicolumn{1}{c}{ Telescope } & \multicolumn{1}{c}{ Filters used } & Band width (in $\AA)$ \\
\hline ESO-WFI & $U_{38}$ & $3100-4000$ \\
VLT-VIMOS & $U_{\text {VIMOS }}$ & $3300-4000$ \\
ACS-HST & $B(F 435 W) V(F 606 W), i(F 775 W), z(F 850 L P)$ & $3400-11000$ \\
VLT-ISAAC & $J_{\text {ISAAC }}, H_{\text {ISAAC }}, K \mathrm{~s}_{\text {ISAAC }}$ & $11000-24000$ \\
Spitzer - IRAC instrument & $3.6 \mu, 4.5 \mu, 5.8 \mu, 8 \mu$ & $30000-100000$ \\
\hline
\end{tabular}

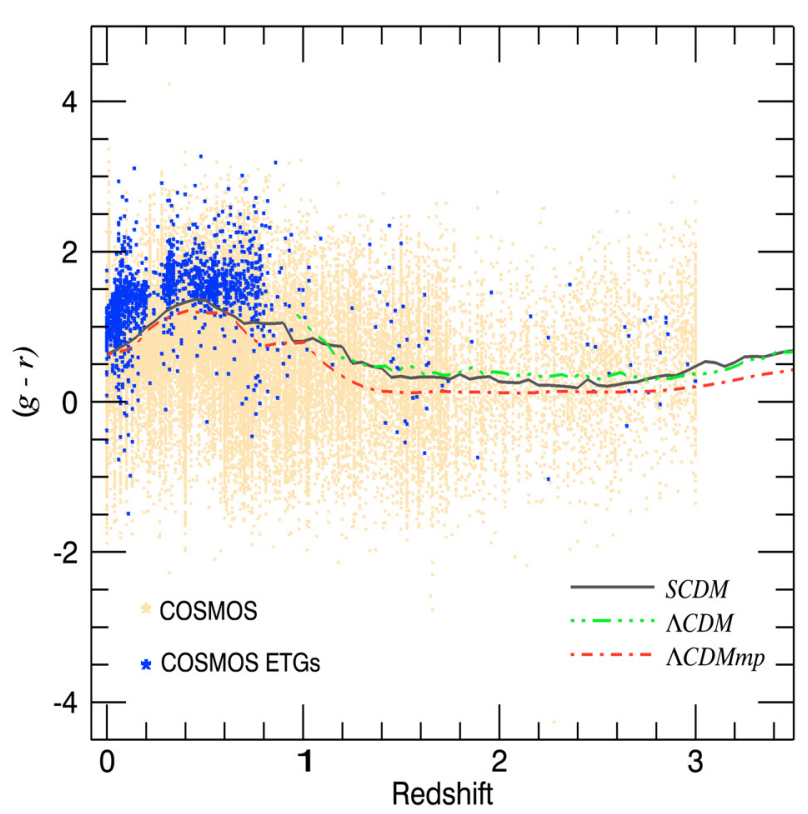

Fig. 19. Same as Fig. 17 but for the $(g-r)$ color (SDSS survey passbands).

\subsection{GOODS database}

For the sake of comparison, we consider now the Great Observatories Origins Deep Survey - GOODS database (Giavalisco et al. 2004). The survey is based on the observations of two separate fields centered on the Hubble Deep Field North (HDFN) and Chandra Deep Field South (CDFS) and includes ultra-deep images from ACS on HST, from mid-IR satellite Spitzer, as well as from a number of ground-based facilities (see Table 5).

Galaxies exhibit a range of morphologies that are difficult to determine automatically, so a manual classification is often used to test the efficacy of automated classifiers. For this reason, in order to select ETGs from the database we have cross-correlated two catalogues: the GOODS - Multiwavelength Southern Infrared Catalogue (GOODS-MUSIC) by Grazian et al. (2006) to determine the redshift and the one by Bundy et al. (2005) to fix the morphology. These are good catalogues to rely on. The first one indeed contains redshifts of high precision. In fact the GOODS-MUSIC database (Grazian et al. 2006) comprises photometric and spectroscopic information for galaxies in the GOODS Southern Field. For these objects they find excellent agreement between photometric and spectroscopic redshifts over the range $0<z<6$ (see Fig. 12 in Grazian et al. 2006 , for the $z_{\text {spec }}-z_{\text {phot }}$ relation).

In the second one, Bundy et al. (2005) present a morphological catalogue of galaxies in the GOODS North and South Fields, for which the morphological classification has been made by hand, therefore more being reliable than the one derived from automated procedure. The study relies on the combination of many

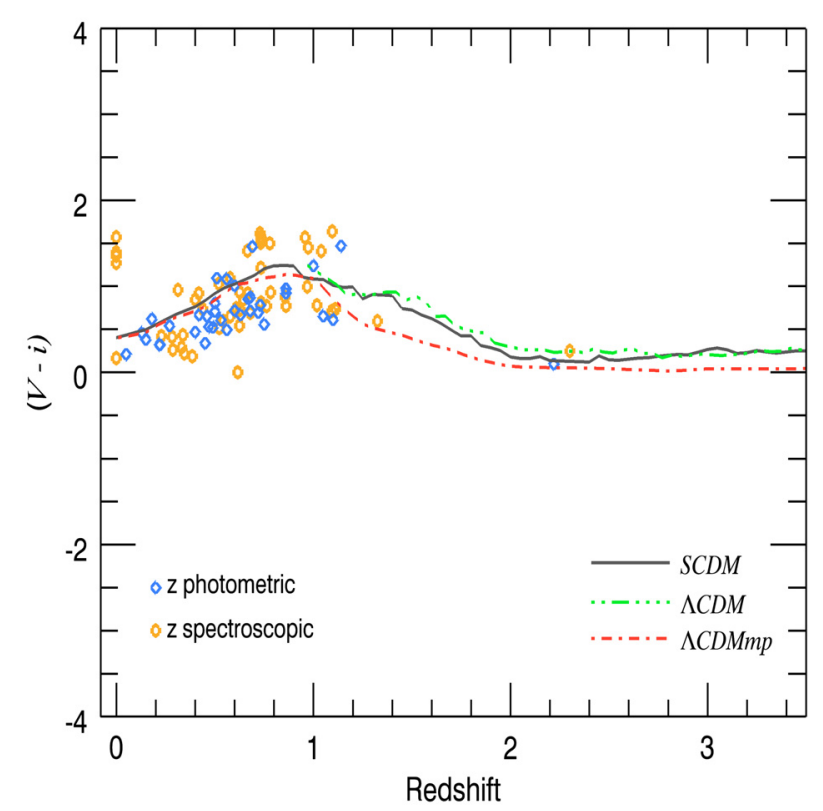

Fig. 20. Cosmological evolution with redshift of the $V(F 606 W)-$ $i(F 775 W)$ colors of the GOODS survey (ACS-HST passbands) for early-type galaxies with spectroscopic (empty circle) and photometric (filled circles) redshift determination as indicated. The galaxy models for the three different cosmological scenarios are shown superimposed to the data, continuous and dotted lines as labelled. The $\Lambda$ CDM case is shown for $z>1$.

different data sets in the GOODS fields including infrared observations as listed in Table 5, spectroscopic and photometric redshifts, and HST morphologies. The catalogue contains objects with a magnitude limit based on HST-ACS imaging data released by the GOODS team (Giavalisco et al. 2004). A $z$-band selected catalogue was constructed running SExtractor (Bertin \& Arnouts 1996) and considering a magnitude limit of $z_{\mathrm{AB}}<22.5$, where reliable visual morphological classification was deemed possible. All magnitudes are defined in the ABmag system and they assume a cosmology with $\Omega_{\mathrm{M}}=0.3, \Omega_{\Lambda}=0.7$, and $H_{0}=70$. The resulting sample of objects over both GOODS fields was inspected visually by Bundy et al. (2005) who classified each one of them, by using the technique discussed in Brinchmann \& Ellis (2000), according to a scale that separates stars from compact objects and galaxies of different morphological type.

We cross-correlated the two catalogues described above to recover a data set of galaxies with reliable morphological classification and precise redshift determination. Therefore these samples of galaxies should be considered much better selected than the sample derived from COSMOS. Standing on those arguments, a final sub-sample of ETGs is selected from the complete database. The total amounts to 118 objects.

In Figs. 20-22, the $(V(F 606 W)-i(F 775 W)),(V(F 606 W)-$ $z(F 850 L P))$, and $\left(V(F 606 W)-K \mathrm{~s}_{\text {ISAAC }}\right)$ colors of this selected sample are shown together with the theoretical predictions. The 


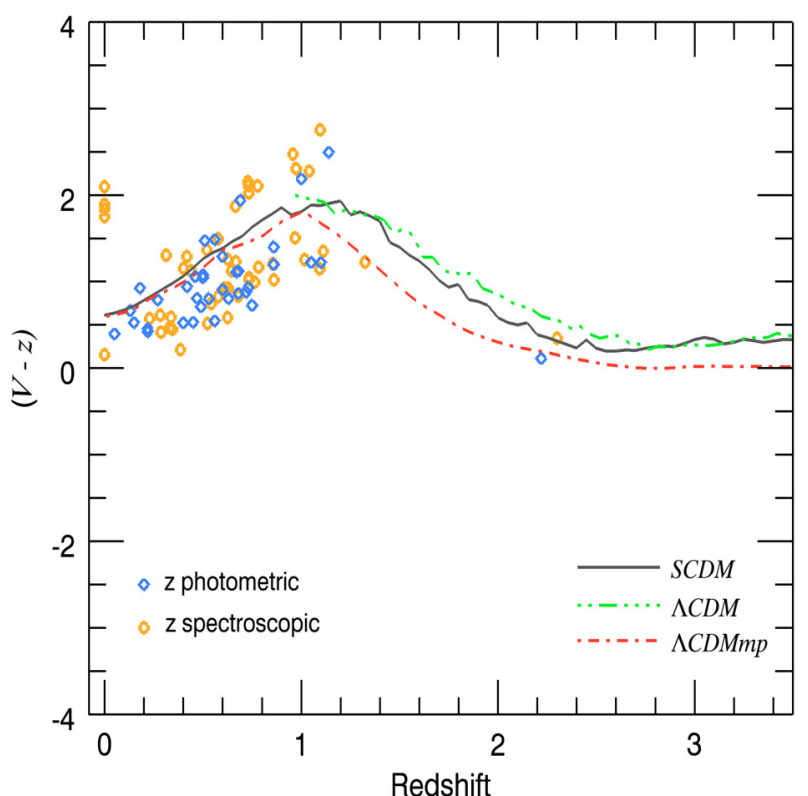

Fig. 21. Same as Fig. 20 but for the $V(F 606 W)-z(F 850 L P)$ colors (ACS-HST passbands).

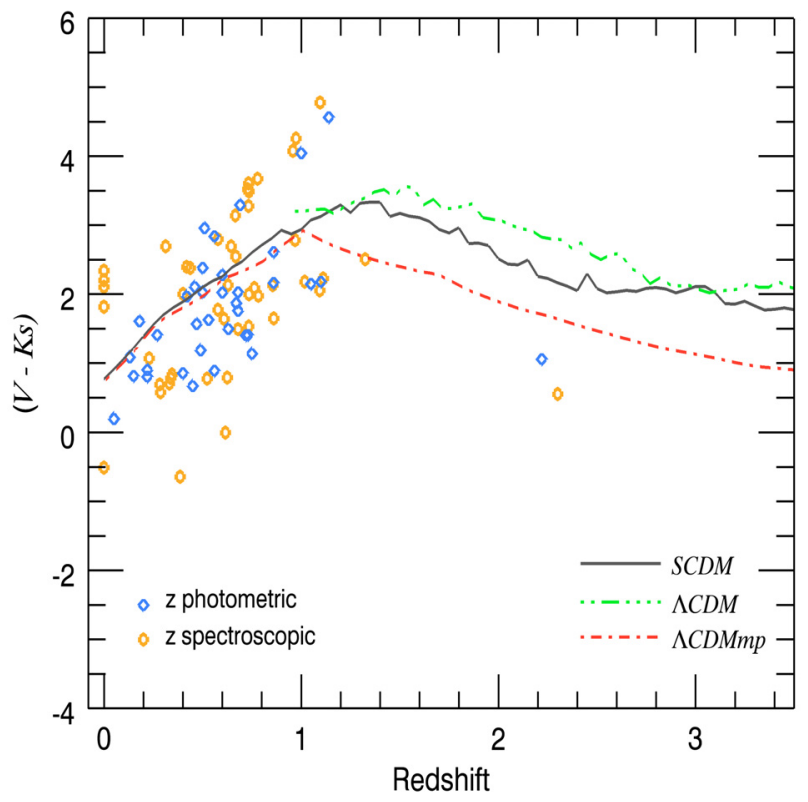

Fig. 22. Same as Fig. 20 but for the $V(F 606 W)-K$ s ISAAC $_{\text {colors }}(V$ passband from ACS-HST and $K \mathrm{~S}_{\text {ISAAC }}$ passband from VLT-ISAAC).

same remark on the redshift spanned by the model $\Lambda \mathrm{CDM}$ made above applies also here. For all of the colors, the agreement between data and theory concerning the photometric, cosmological evolution with redshift is remarkably good. Furthermore, the theoretical colors seem to match the GOODS data much better than COSMOS values.

This clearly shows that the reliability of the morphology classifier plays an important role in these matters. The selection in GOODS is made by correlating two catalogues, one containing photometric and spectroscopic redshifts, the other listing galaxies with good morphological classification (made by hand). In contrast, in COSMOS classification and selection are fully automated with no any cross-correlation with other criteria.

Another possible explanation for the differences between the results from COSMOS and GOODS, could be an intrinsic difference in the samples of observed galaxies. First, the COSMOS database has a lower magnitude limit that makes the morphological classification more difficult. Second, two different packages are used to calculate the redshift with a mean dispersion of 0.0315 . Finally, there are some problems with the using the SExtractor (Bertin \& Arnouts 1996) star/galaxy separator parameter that is not stable with a variable seeing. All this goes in favor of the GOODS database of ETGs.

\section{Surface photometry of ETGs}

Surface photometry is one of the most powerful tools to study the properties and history of ETGs. The analysis is based on fitting ellipses to the isophotes of a galaxy. The oldest fit of the radial intensity profile is the empirical de Vaucouleurs law (de Vaucouleurs 1948) that has now been extended by the more flexible Sérsic profile (Sérsic 1968). The derived properties, of which the most important ones are the intensity distribution, the radial ellipticity, and the position angle profiles, provide basic information such as effective radius, deviations from the ellipses, isophote twisting, triaxiality, and absolute magnitudes. The associated higher order Fourier coefficients from the fits reveal the intrinsic "boxy" or "disky" appearance of the isophotes which can be used to uncover the underlying stellar components.

In this section we will show how, starting from a 3D numerical simulation of a galaxy, we can recover "artificial" images projected on a plane, from which photometric and structural parameters, such as luminosity, magnitudes, colors, and effective radius, can be calculated. These images are analyzed as if they were realistic images of galaxies taken with a telescope. In this way we can derive morphological and structural parameters of the models that can be compared with those of real ETGs. In particular we derive the Kormendy relation (the projection of the Fundamental Plane on the luminosity-radius plane). Finally, we make use of the SDSS photometric system.

For the aims of this analysis, we did not include the effects of extinction. This can be justified as follows. The artificial images we are using refer to the present epoch, when SFR has dropped by orders of magnitudes with respect to the past and the galaxies are nearly passively evolving. Furthermore, as a consequence of galactic winds, all interstellar gas and dust have been expelled from the central regions of galaxies. The issue has been discussed in some detail by Galletta et al. (2007) who made use of the same galaxy models and to whom we refer. In brief, to investigate the spectro-photometric evolution of the NB-TSPH models by Merlin \& Chiosi (2006, 2007), Galletta et al. (2007) studied the relative spatial distribution of stars and gas and found that while most of the stars are located inside about half viral radius, most of the gas falls outside. This implies that effects of dust (extinction) become important only in the very outer regions of ETGs. Of course, a completely different situation is expected to occur at high redshifts, when the evolutionary stage reached by a galaxy is such that most of gas is likely to fall in the innermost regions whereby star formation is still active. In such a case, the effects of extinctions cannot be neglected. In any case, work is in progress to include extinction in the SEDs of simulations as observed along a given line of sight, by considering the geometrical structure of the galaxies.

\subsection{Artificial images}

Starting from the 3D NB-TSPH simulations, we construct 2D images projecting the volume with $|z|<100 \mathrm{kpc}$ onto the $x y$ plane. The information we need are the spatial coordinates 

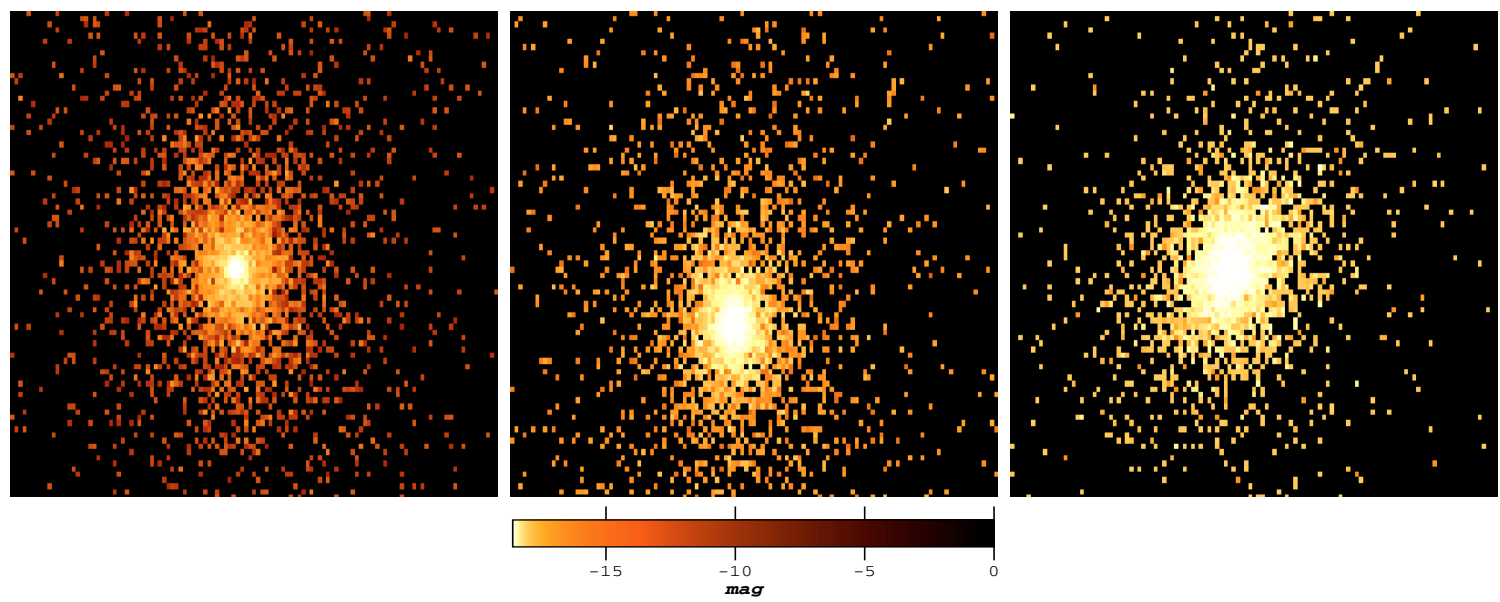

Fig. 23. $r$-band artificial image of a $80 \times 80 \mathrm{kpc}^{2}$ region with a $200 \times 200$ mesh points for the last age of the evolution of the model galaxies: $\mathrm{SCDM}$ left, $\Lambda \mathrm{CDM}$ middle, $\Lambda \mathrm{CDM}_{\mathrm{mp}}$ right.

Table 6. Construction of artificial images: dimensions and grid scales.

\begin{tabular}{cccccc}
\hline \hline & $40 \mathrm{kpc}$ & $60 \mathrm{kpc}$ & $80 \mathrm{kpc}$ & $100 \mathrm{kpc}$ & $200 \mathrm{kpc}$ \\
\hline $50 \mathrm{gp}$ & $0.8 \frac{\mathrm{kpc}}{g p}$ & 1.2 & 1.6 & 2.0 & 4.0 \\
$100 \mathrm{gp}$ & 0.4 & 0.6 & 0.8 & 1.0 & 2.0 \\
$200 \mathrm{gp}$ & 0.2 & 0.3 & 0.4 & 0.5 & 1.0 \\
$500 \mathrm{gp}$ & 0.08 & 0.12 & 0.16 & 0.2 & 0.4 \\
\hline
\end{tabular}

of the star-particles and their SEDs. With these projections on a plane we construct discrete grids at the nodes of which we calculate the total flux given by all the particles along the line of sight. These fluxes feed the photometric code to compute the magnitude and/or color in a given band for each mesh point of the grid. The flux of each star-particle of given age and metallicity is the one of the associated SSPs. Therefore there is full consistency between the SEDs of the star particles and those required by the photometric code in use.

To create good artificial images, the grids must be large enough to encompass all the star-particles of the model galaxy, and must contain a large enough number of grid points to eventually get smooth images. A scale length of $80-100 \mathrm{kpc}$ is long enough to include all the star-particles of our simulations and a grid with 100-200 points along each direction is good enough to secure smooth images; the best combination to obtain satisfactory results is a scale of 0.4 to $0.8 \mathrm{kpc}$ per grid point and direction. Table 6 gives the resolution under which images have been created: the choice of the scale depends on the model galaxy and its diameter.

Images are built up for the last computed stage of the model galaxies at the ages listed in Table 3 . Figure 23 shows the $2 \mathrm{D}$ distribution of the star-particles of the models as traced by the star-particle $r$-magnitudes of the SDSS system. A grid of $200 \times$ 200 mesh points is used to span a square region of $80 \mathrm{kpc}$ side. In all the models, the old star-particles appear to be spatially distributed mimicking the morphology of an elliptical galaxy. The left panel refers to the SCDM model, the middle to the $\Lambda$ CDM, and the right to the $\Lambda C D M_{m p}$. In displaying these frames the intensity contrast between the bright central regions and the low surface brightness of the outer parts is very difficult to portray using a linear relation, so that the logarithmic scale is more convenient. It is worth noting that the inclination of the major axis of the projected distribution with respect to the coordinate axes simply reflect the spatial distribution of the star-particles in the
$3 \mathrm{D}$ space and in the $2 \mathrm{D}$ projection. No other physical meaning in involved.

\subsection{Isophotal analysis}

The images used in this section are similar to those of Fig. 23 but are based on a grid of $100 \times 100$ mesh points (enough to describe a square region of $80 \mathrm{kpc}$ side). These images are analysed with the IRAF packages Stsdas.Isophot (designed to deal with elliptical isophotes) and Ellipse (designed to derive the brightness distribution of a galaxy).

Stsdas.Isophot (using all the data of the galaxy image) extracts the intensity levels, which are nicely fitted by ellipses, and determines the center, the ellipticity, the position angle, and the length of the semi-major axis. However, small but significant deviations from pure ellipses are also present. For each isophote, these deviations are measured along the contour and are recovered by the Fourier analysis on the distance of the ellipsoidal contour as a function of the azimuthal angle.

Ellipse fits the elliptical isophotes and measures the isophotal parameters of a galaxy's image in whatsoever passband (SDSS in our case). The parameters are determined for each isophote. The algorithm follows the method described by Jedrzejewski (1987). In brief, assumed a provisional guess for an isophote's center coordinates $\left(X_{\mathrm{c}}, Y_{\mathrm{c}}\right)$, ellipticity $(\epsilon)$ and semi-major axis position angle $(\phi)$, at each point along the semi-major axis, the intensity $I(\phi)$ of the image is azimuthally sampled along an elliptical path. $I(\phi)$ is then expanded into a Fourier series as

$I(\phi)=I_{0}+\sum_{k}\left[A_{k} \sin (k \phi)+B_{k} \cos (k \phi)\right]$

A best fit procedure (minimization of the sum of the squares of the residuals between the real distribution of data and an elliptical one) fixes the parameters $X_{\mathrm{c}}, Y_{\mathrm{c}}, \epsilon$, and $\theta$. The expansion is truncated at the first two moments that are sufficient to describe an ellipse. The intensity profile as a function of the position angle is then fitted by the weighted least-squares

$I=I_{0}+A_{1} \sin (\phi)+B_{1} \cos (\phi)+A_{2} \sin (2 \phi)+B_{2} \cos (2 \phi)$.

The amplitudes $A_{1}, B_{1}, A_{2}, B_{2}$ give information on how much the estimated intensity profile deviates from the real one, so they give the errors in the fitting procedure. The image data is fitted by the function

$I=I_{0}+A_{3} \sin (3 \phi)+B_{3} \cos (3 \phi)+A_{4} \sin (4 \phi)+B_{4} \cos (4 \phi)$ 
where the amplitudes $A_{3}, B_{3}, A_{4}, B_{4}$ measure the isophote's deviations from perfect ellipticity.

Higher order moments $(k \geq 3)$ define deviations of the isophotes from ellipses. In practice, moments beyond the fourth cannot be measured accurately; third and fourth-order moments are calculated from the equation above by fixing the first and second-order moments to their best-fit values. The third-order moments $\left(A_{3}\right.$ and $\left.B_{3}\right)$ represent isophotes with three-fold deviations from ellipses (e.g., egg-shaped or heart-shaped) while the fourth-order moments $\left(A_{4}\right.$ and $\left.B_{4}\right)$ represent four-fold deviations. Rhomboidal or diamond-shaped isophotes translate into a non-zero $A_{4}$. For galaxies which are not distorted by interactions, $B_{4}$ is the most meaningful moment: a positive $B_{4}$ indicates "disky" isophotes (i.e., with semi-major axis $B_{4} \times 100$ percent longer than the best fitting ellipse), whereas a negative $B_{4}$ indicates "boxy" isophotes (i.e., with semi-major axis $B_{4} \times 100$ percent shorter than the best fitting ellipse Jedrzejewski 1987).

When running Ellipse on model galaxies, we adopt the following guidelines. The length of the semi-major axis is increased on a logarithmic scale (10\% longer in our case). With a new semi-major axis, a new isophote is calculated using the bestfit parameters obtained from the previous isophote. In general, all the parameters can vary freely, even though the routine may fail to converge on isophotes with large deviations from ellipses. In such cases to achieve convergence it is necessary to fix the value of one or more parameters. The isophotal center is not let wander around by more than 2 grid points between consecutive isophotes; in practice the center is found to be rather stable.

Given the isophotal center and the semi-major axis, at any position $a$ along the semi-major axis we determine the radius, $r_{\mathrm{gm}}$, of a circle corresponding to the local ellipse as

$r_{\mathrm{geo}}=a \sqrt{1-\epsilon(a)}$

where $\epsilon(a)$ is the ellipticity at the position $a$. This is equivalent to take the geometric mean of the local semi-major and semiminor axes. In this way all the profiles are reduced to equivalent circular profiles and the integrated intensity is measured within this equivalent circle. With this procedure we recover, as a function of the semi-major axis length, the radial profiles of the mean isophotal intensity, ellipticity, position angle, local radial intensity gradient, mean isophotal magnitude, 3rd and 4th order deviations from ellipse. The errors on intensity, magnitude and gradient are obtained from the rms scatter of the intensity along the fitted ellipse. The errors on the geometrical parameters are obtained from the internal errors in the harmonic fit, after removal of the first and second fitted harmonics. Errors on the harmonic amplitudes are obtained from the fit error after removal of all the harmonics up to including the one being considered.

After using the ellipse task to measure the mean radial intensity profiles and fit ellipses to the image, the program Bimodel is used to reconstruct a model image from the results of isophotal analysis. Bimodel creates a 2D smooth image of the source image. In Fig. 24 are shown the smooth images of the three simulations corresponding to the discrete images of Fig. 23 with the elliptical isophotes overlapped. The same remark made for the inclination of the semi-major axis with respect to the $X$-axis in Fig. 23 applies also here.

The 3rd and 4th harmonics from the photometry are added to the model. This option is most useful when working close to the central intensity peak. As explained in Jedrzejewski (1987), the sampling at small radii may introduce a "boxy" component. More accurate modelling of the central region is generally achieved when including this component.
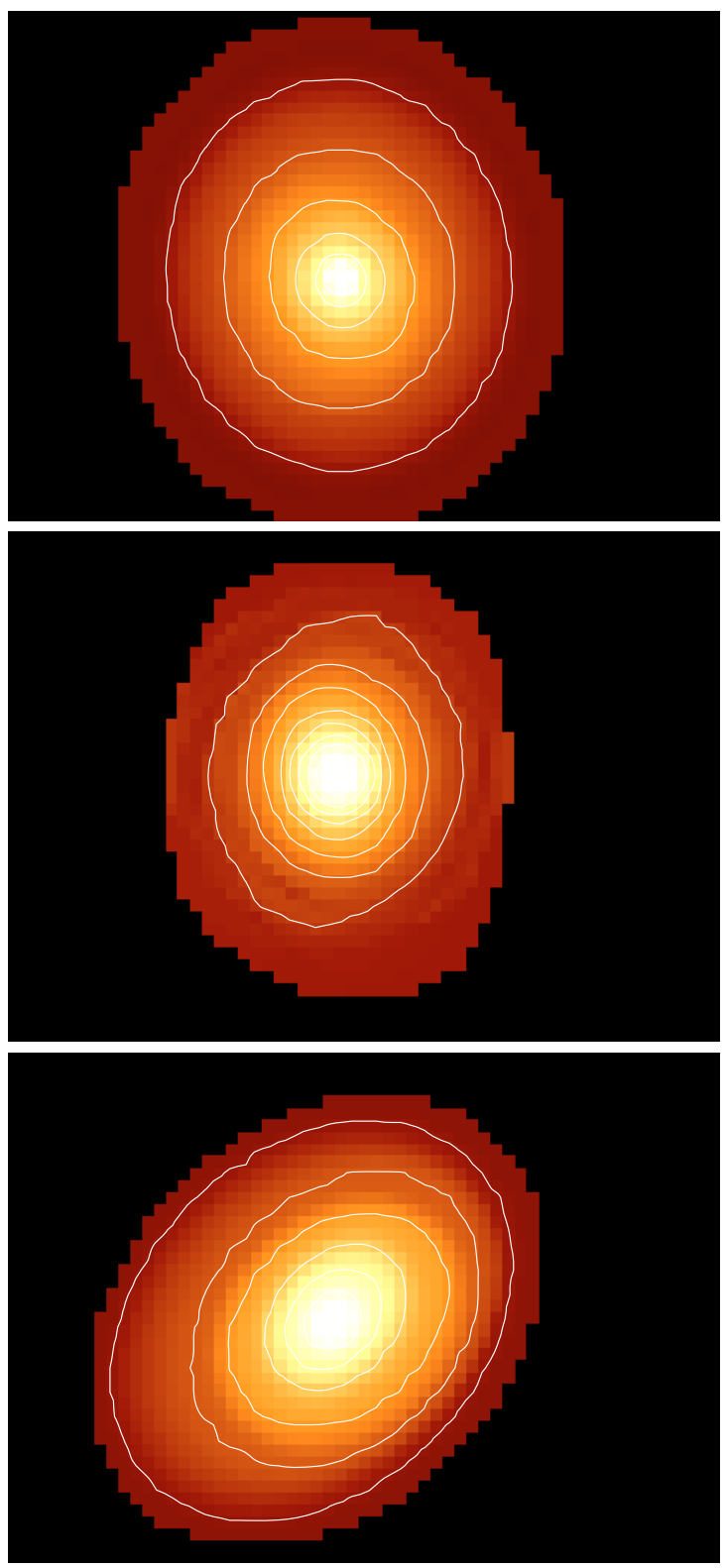

Fig. 24. Two-dimensional models of the optical $r$-band magnitude in the $x y$-plane of the images shown in Fig. 23. Superposed are elliptical isophotes (white solid lines). The top panel is for SCDM, the middle panel for $\Lambda \mathrm{CDM}$, and the bottom panel for $\Lambda \mathrm{CDM}_{\mathrm{mp}}$.

\subsection{Parametrization of the intensity profile}

The intensity $I(R)$ and corresponding surface brightness $\mu(R)$ are given by the Sérsic laws

$$
\begin{aligned}
& I(R)=I_{\mathrm{e}} \exp \left\{-b_{n}\left[\left(\frac{R}{R_{\mathrm{e}}}\right)^{1 / n}-1\right]\right\} \\
& \mu(R)=\mu_{\mathrm{e}}-2.5 b_{n}\left[\left(\frac{R}{R_{\mathrm{e}}}\right)^{1 / n}-1\right] \log e
\end{aligned}
$$

where $r$ is the radius from the center in $\mathrm{kpc}, R_{\mathrm{e}}$ is the effective radius, and $I_{\mathrm{e}}$ and $\mu_{\mathrm{e}}$ are the effective intensity and effective surface brightness, respectively, within $R_{\mathrm{e}} . b_{n}$ is a positive parameter that, for a given $n$, can be determined from the definition of $R_{\mathrm{e}}$ and $\mu_{\mathrm{e}}$. 
Table 7. Sérsic indexes and effective radii expressed in kpc for the SDSS photometric passbands and for all model galaxies.

\begin{tabular}{ccccccc}
\hline \hline & \multicolumn{2}{c}{ SCDM } & \multicolumn{2}{c}{$\Lambda$ CDM } & \multicolumn{2}{c}{$\Lambda \mathrm{CDM}_{\mathrm{mp}}$} \\
\hline band & $n$ & $R_{\mathrm{e}}$ & $n$ & $R_{\mathrm{e}}$ & $n$ & $R_{\mathrm{e}}$ \\
\hline$u$ & 3.22 & 6.42 & 2.2 & 2.38 & 3.97 & 4.31 \\
$g$ & 3.08 & 5.78 & 2.1 & 2.43 & 3.69 & 3.67 \\
$r$ & 3.07 & 5.42 & 1.87 & 2.7 & 3.37 & 3.27 \\
$i$ & 3.86 & 5.83 & 1.99 & 2.73 & 3.14 & 3.07 \\
$z$ & 4.42 & 5.98 & 2.21 & 2.6 & 2.94 & 2.95 \\
\hline
\end{tabular}

For a Sérsic model with $1 \lesssim n \lesssim 10$, the effective radius $R_{\mathrm{e}}$ contains roughly half the integrated light if $b_{n}=2 n-0.324$ (Trujillo et al. 2001). The parameter $n$ controls the overall shape of a Sérsic profile, with low $n$ values producing curved profiles with logarithmic slopes which are shallow in the inner regions, and steep in the outer parts, while high $n$ values produce extended profiles with less overall curvature.

By definition, the parameters are chosen in such a way that the model reduces to the de Vaucouleurs law for $n=4$, and the consistency with the usual interpretation of $R_{\mathrm{e}}$ as the radius enclosing half-light is secured.

The Sérsic model offers significant advantages. First and foremost, it provides a good description of the inner (100 pc scale) profiles, and a significantly better description when the profiles are extended to the kpc-scale (Graham et al. 2003; Trujillo et al. 2004).

The intensity profile is derived using the nfitld algorithm which provides $1 \mathrm{D}$, non-linear fits to the image (chi-square minimization). As nfitld supports any analytical fitting function, given the initial guesses for the function coefficients, we have obtained the intensity profile of the model galaxy with a Sérsic law with suitable index $n$, together with the effective radius $R_{\mathrm{e}}$ and the effective intensity $I_{\mathrm{e}}$. Figure 25 shows the best fitting Sérsic function for the $r$-band in the particular sample. The profile inside the central region whose radius is equal to $1-2$ times the softening length (1-2 kpc in our case), is excluded from the fit as it is likely to be influenced by force softening. To derive the effective radius, we reach large enough distances such as 15-20 kpc. The solid curve represents the best-fit Sérsic model to the final profile (shown as solid symbols).

From the fitted intensity profiles and the best-fit parameters, we calculate the effective radii $R_{\mathrm{e}}$ and the total magnitudes in all bands for the three galaxy models. The Sérsic index and $R_{\mathrm{e}}$ are listed in Table 7 for the various passbands.

The effective radius varies with the band from which is derived, roughly decreasing as the band moves toward the shorter wavelengths. Bernardi et al. (2003a) finds that half-light angular sizes of the galaxies in their sample are indeed larger in the blue bands and they show how the effective physical radii in their sample changes in the four ${ }^{1}$ bands. This trend is followed by our simulations: the agreement is particularly good for the $\Lambda \mathrm{CDM}_{\mathrm{mp}}$ model. For the other two, the trend is recovered only in the bluer bands, whereas the radius increases again as the band shifts toward longer wavelengths.

\subsection{Structural properties}

In the various panels of Fig. 26 we show the basic structural parameters of the three models. In brief, the ellipticity $\epsilon$ (top

\footnotetext{
1 They exclude the $u$-band as the measurements are affected by errors larger than the others.
}

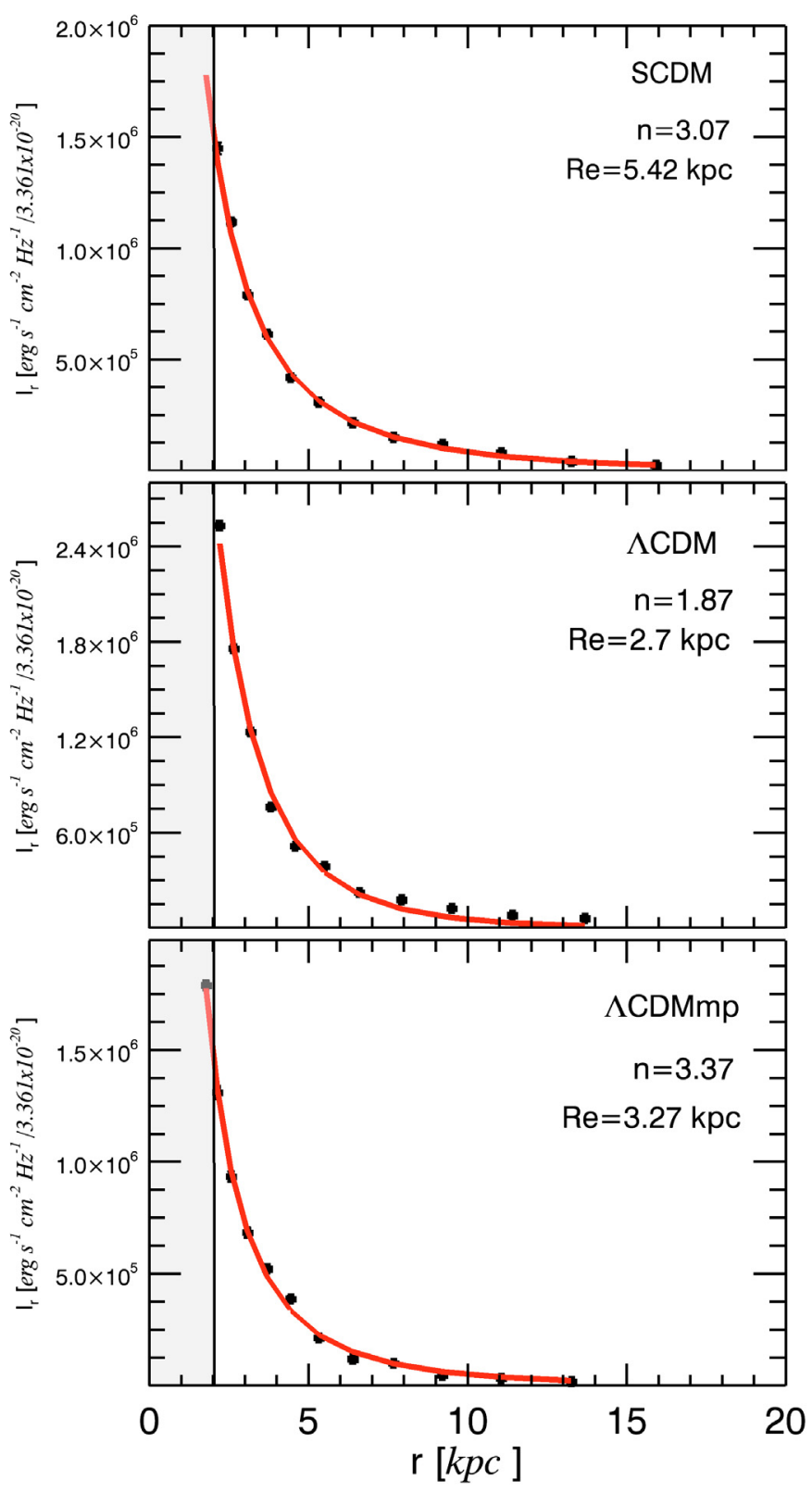

Fig. 25. Intensity profiles fitted with a Sérsic law of variable index. The $r$-band Sérsic index $n$ and the effective radius $R_{\mathrm{e}}$ are specified in the panel for each models. We exclude the central region within $R \sim 1-2 \mathrm{kpc}$ (shaded area), because the intensity profile is smeared out by the gravitational softening technique.

panels), position angle PA (middle panels), and deviation $A_{4}$ of the isophotes from pure ellipses (bottom panels) are shown as a function of the "geometric mean" radius $r_{\mathrm{gm}}$, as defined in Eq. (20) for all the galaxy models as indicated. To the sake of illustration, we show the results limited to the $r$-band image.

Although the isophotes shown in Fig. 24 are well approximated by ellipses, small but significant deviations from perfect ellipsoidal shapes are measured. Of particular interest is the $A_{4}$ parameter ${ }^{2}$, which measures the deviations from perfect ellipses: $A_{4}<0$ corresponds to "boxy" isophotes, whereas $A_{4}>0$ implies "disky" isophotes.

\footnotetext{
${ }^{2} A_{4}$ corresponds to the fourth order moment $B_{4}$ of Eq. (19).
} 
R. Tantalo et al.: Spectro-photometric models of early type galaxies
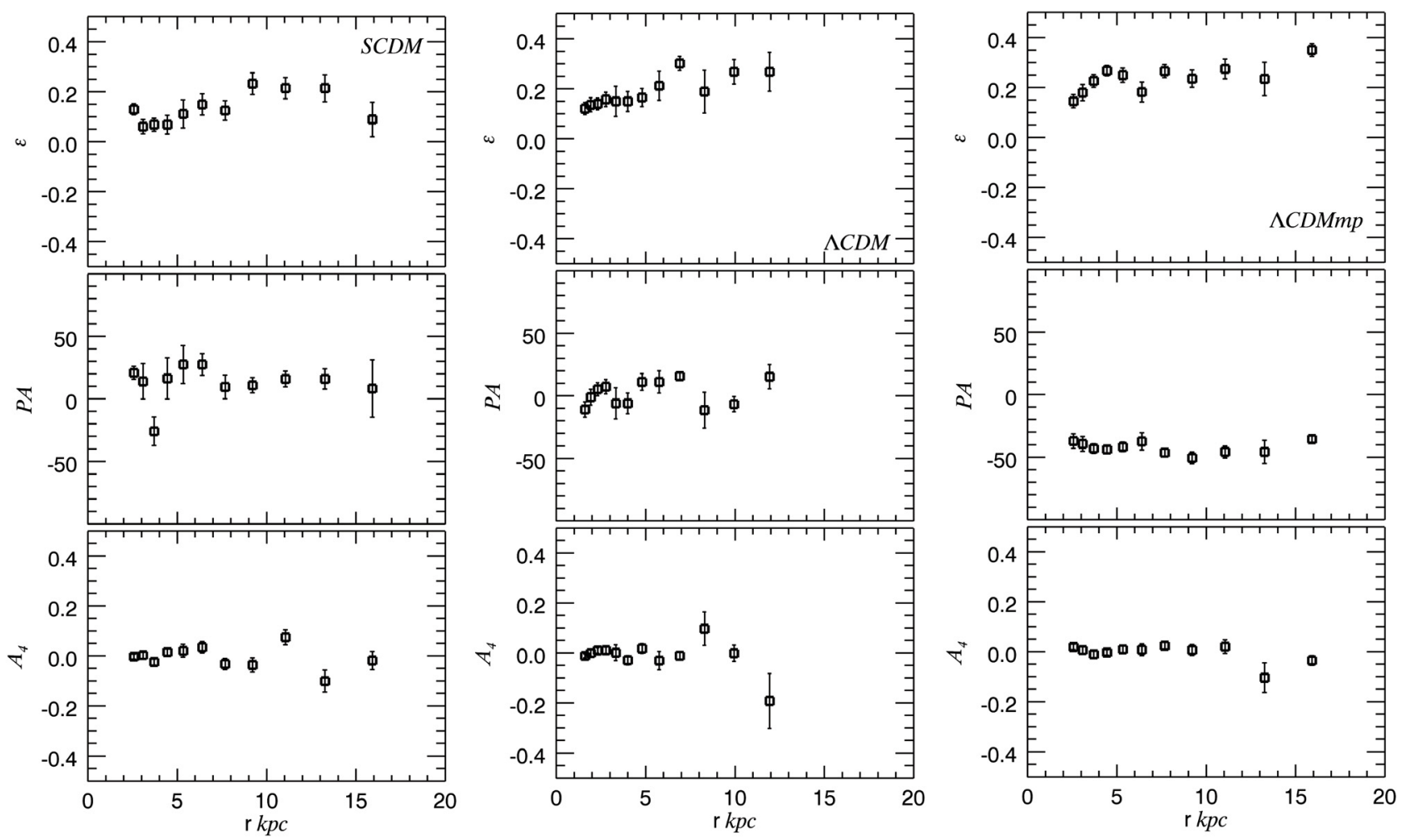

Fig. 26. Ellipticity $\epsilon$, position angle PA, and $A_{4}$ radial profiles for the three models as indicated.

The radial profile of the $A_{4}$ parameter indicates that the deviations from perfect ellipses are generally negligibly small for the $\mathrm{SCDM}$ and $\Lambda \mathrm{CDM}_{\mathrm{mp}}$ models. In the case of the $\Lambda \mathrm{CDM}$ model, the negative values of $A_{4}$ in the outer regions tell us that there is a "boxy" structure.

\subsection{Color profiles}

As mentioned before, Bernardi et al. (2003a) found that halflight angular sizes of the galaxies change in function of the band in which they are measured, finding larger radii with bluer bands. On average, the fact that ETGs have this trend implies that they present color gradients. It is known from observations that these gradients are such that ETGs are redder in their cores and bluer in the outskirts. This fact is thought to originate from variations in age or metallicity of the underlying stellar populations (Worthey et al. 1994; Tantalo et al. 1998a).

The radial color profiles of our models are shown in the three panels of Fig. 27 which display the $u-g, g-r, r-i$, and $i-z$ color profiles in the SDSS photometric system. With the physical processes we have considered, colors are bluer in the central cores and the color gradients are very small for the SCDM and $\Lambda$ CDM models (left and middle panels of Figs. 27). In contrast, the central regions are redder and the color gradients are significant for the $\Lambda \mathrm{CDM}_{\mathrm{mp}}$ model (right panel of Fig. 27). Therefore, the typical color gradients of ETGs are not strictly reproduced by our models, except for the $\Lambda \mathrm{CDM}_{\mathrm{mp}}$ case.

The main reason for the central regions being bluer than expected and observed resides in the prolonged stellar activity present mainly in the central regions of all the models together with insufficient increase of the mean metallicity in those regions. To cast light on the effect of the long tail of star formation, we have artificially stopped star formation at different epochs. The details of these experiments are not shown here for the sake of brevity. As expected, stopping star formations immediately yields redder colors in the center, for instance by about $0.1 \mathrm{mag}$ in $(B-V)$. In addition, we have applied the cut in star formation to star-particles enclosed within a given galacto-centric distance going from $50 \mathrm{kpc}$ to $R_{\mathrm{e}} / 2$. The result is that the effect on the color gets stronger moving outward. In all cases, the color difference amounts to 0.1 to $0.2 \mathrm{mag}$ in the typical $(B-V)$. What we learn from this is that further investigation of other star formation prescriptions and effects of feedback with better resolution in the central regions is required.

\section{Scaling laws: the Kormendy relation}

As long known, ETGs are similar in their structural and dynamical properties and obey to empirically relationships among colors, luminosities, half-light radii, surface brightness profiles, and velocity dispersions that are ultimately related to their stellar content and dynamics. They are known as the Scaling Laws, among which particularly important are the Fundamental Plane and the Kormendy Relation.

Fundamental Plane (FP). Long ago Djorgovski \& Davis (1987) and Dressler et al. (1987) found that

$\log R_{\mathrm{e}}=\alpha \log \sigma_{0}+\beta<\mu>_{\mathrm{e}}+\gamma$

where $R_{\mathrm{e}}$ is the effective radius, $\sigma_{0}$ the central velocity dispersion, $\langle\mu\rangle_{\mathrm{e}}$ the mean surface brightness within the effective radius $\left(\langle\mu\rangle_{\mathrm{e}}=-2.5 \log \left\langle I_{\mathrm{e}}\right\rangle+\right.$ const. $)$, and $I_{\mathrm{e}}$ is the effective luminosity. The coefficients change with the passband in use. Typical values for the Johnson $B$-band are: $\alpha=1.25, \beta=0.32$, and $\gamma=-8.895$ (Bender et al. 1998). A remarkable property is the variation of the coefficient $\alpha$ in the different filter passbands, and the almost constant value of $\beta$ (Bernardi et al. 2003c). 

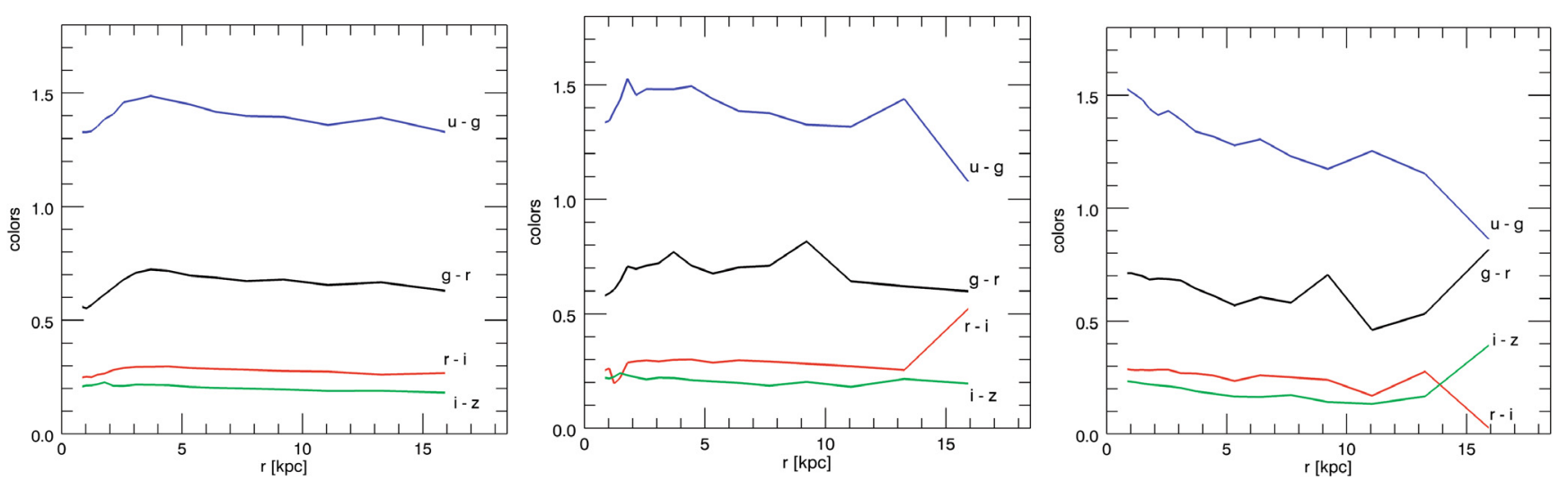

Fig. 27. Left panel: radial profiles of the SDSS colors for the SCDM model derived from the simulated image $100 \times 100$ grid points shown in Fig. 23. Middle panel: the same as in the left panel but for the $\Lambda \mathrm{CDM}$ model. Right panel: the same as in the previous panel but for the $\Lambda \mathrm{CDM}_{\mathrm{mp}}$ model.

The existence of the FP has strong implications on galaxy formation and evolution theories. The small scatter in the FP (see e.g. Jorgensen et al. 1996) and its apparent lack of evolution with redshift (Franx 1995; van Dokkum \& Franx 1996; Bender 1996; Ellis et al. 1997; van Dokkum et al. 2000), the homogeneity (Bower et al. 1992a), and the evidence for short ( $<1$ Gyr) star formation timescales in these systems, all indicate that the bulk of stellar population in ETGs indeed formed at high redshift $(z>2)$. Measuring the three parameters entering the FP for ETGs at varying redshift highlights some important questions concerning their age, formation history, and internal properties. One can answer questions such as how far in the past does the FP apply and whether its parameters evolved significantly with time. A study of the galaxy properties as a function of look-back time provides a good probe of the possible evolutionary differences. In this sense, we plan to study this scaling law at intermediate redshift by deriving its key parameters as function of time, i.e. considering the evolution with redshift of the ETGs.

In order to compare our models with real galaxies on the FP, we need the theoretical central velocity dispersion, $\sigma_{0}$. Observationally, this is evaluated within an aperture typically less than about half the effective radius. Unfortunately, velocities within such small radii in the simulations are significantly affected by the softening parameter that in our case amounts to $1 \mathrm{kpc}$. As accurate predictions for $\sigma_{0}$ are not possible with our models, we have to leave aside the analysis of the FP.

Kormendy Relation (KR). Better chances are possible with this relation. The KR is the projection of the FP onto the luminosityradius plane. It relates $\langle\mu\rangle_{\mathrm{e}}$ to $R_{\mathrm{e}}$. Once the dependence on $I_{\mathrm{e}}$ is made explicit we get

$R_{\mathrm{e}} \propto I_{\mathrm{e}}^{-0.83}$.

Many studies have confirmed that the luminous ETGs in clusters approximately follow the relation $\langle\mu\rangle_{\mathrm{e}}=\log R_{\mathrm{e}}+$ const. found by Kormendy with slope of $\sim 3$ and intrinsic scatter of $0.3-0.4$.

The KR for galaxies in clusters at increasing redshift has been claimed to be consistent with passively evolving stellar populations (Bower et al. 1992b; Aragon-Salamanca et al. 1993; Bender et al. 1996; van Dokkum \& Franx 1996; Jorgensen \& Hjorth 1997; Ziegler \& Bender 1997; Bender et al. 1998; van Dokkum et al. 1998). On the other hand, some studies have also claimed that the data are consistent with the hierarchical evolutionary scenario (White \& Rees 1978).
La Barbera et al. (2003), working with cluster ETGs at different redshift, found that the slope of the KR is almost invariant up to $z \sim 0.64$ with value of $\sim 2.91 \pm 0.08$. The homogeneity and the invariance with redshift of these distributions is also suggested by the analysis of the SDSS data by Bernardi et al. (2003b).

To compare our models with observational data, we have chosen a sub-sample of ETGs from the Sloan Digital Sky Survey (SDSS; York et al. 2000; Stoughton et al. 2002) database. The SDSS survey has mapped one-quarter of the entire sky, producing a detailed image of it and determining the positions and photometric properties of more than 100 million celestial objects. The SDSS obtained high-resolution images in five different bands, namely $u, g, r, i$, and $z$ (Fukugita et al. 1996), thus allowing for a reliable identification of ETGs and precise measurements of their photometric properties.

Galaxies can be selected using automated pipelines that isolate objects on the basis of their $2 \mathrm{D}$ light distributions. We consider ETGs from the DR2 release selected following the criteria described in Bernardi et al. (2003a) who have produced a catalogue of $~ 9000$ low-redshift ETGs, selected using a combination of SDSS pipeline parameters. This catalogue contains galaxies with a high $i$-band concentration index $\left(r_{50} / r_{90}\right)>2.5$ and in which a de Vaucouleurs (1948) fit to the surface brightness profile is significantly more likely than an exponential fit. Details of the selection can be found in Bernardi et al. (2003a).

In Fig. 28 we show the $M_{r}-R_{\mathrm{e}}$ relation found by Bernardi et al. (2003c) for the sample of elliptical galaxies selected as mentioned above. The luminosity-size relation for $r$-band is $R_{\mathrm{e}} \propto$ $I^{-0.75 \pm 0.01}$. For comparison, we show the results for our three model galaxies. The $R_{\mathrm{e}}$ and $M_{r}$ magnitude within it of the models are: $R_{\mathrm{e}}=5.42 \mathrm{kpc}$ and $M_{r}=-21.77$ for the SCDM model; $R_{\mathrm{e}}=2.7 \mathrm{kpc}$ and $M_{r}=-22.02$ for the $\Lambda \mathrm{CDM}$ case; $R_{\mathrm{e}}=3.27$ and $M_{r}=-20.48$ for the $\Lambda \mathrm{CDM}_{\mathrm{mp}}$ galaxy.

The SCDM and $\Lambda \mathrm{CDM}_{\mathrm{mp}}$ lie above the mean relation but fall in the data crowd. The $\Lambda \mathrm{CDM}$ model falls below the mean line but still compatible with data. No case lies close to the mean line. There are several reasons to account for the marginal discrepancy. The discussion is slightly different for the SCDM and $\Lambda \mathrm{CDM}_{\mathrm{mp}}$ models evolved up to the age of $13 \mathrm{Gyr}$ and the $\Lambda \mathrm{CDM}$ stopped at $7 \mathrm{Gyr}$.

To bring the position of the SCDM and $\Lambda \mathrm{CDM}_{\mathrm{mp}}$ models down to the mean line one should increase the total star mass and $R_{\mathrm{e}}$ by a factor of 1.5 or so. The solution is viable in the sense that other models of the same type with better tuned parameters could reach the agreement. The same reasoning cannot 


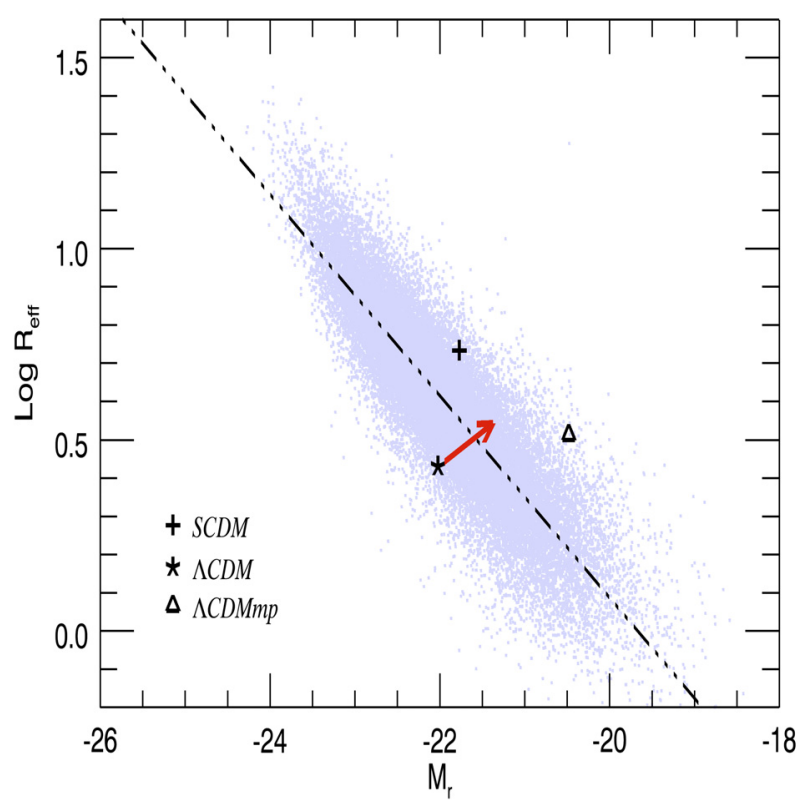

Fig. 28. Magnitude-radius relation in the $r$-band for the ETGs selected from the SDSS DR2 database following the criteria from Bernardi et al. (2003a). Results for our three model galaxies are shown for comparison. See the text for all details.

be applied to the model $\Lambda$ CDM because its evolution terminated at $7 \mathrm{Gyr}(z \sim 1)$. Looking at the fading lines (magnitudes versus age) of Fig. 6 a shift of the $M_{r}$ magnitude of about +1 mag in $6 \mathrm{Gyr}$ (to get the age of $13 \mathrm{Gyr}$ ) is possible without changing the star mass, thus bringing the model onto the mean line without changing $R_{\mathrm{e}}$. Most likely, an increase of $R_{\mathrm{e}}$ of the same entity as in the previous cases is also possible. The arrow in Fig. 28 shows the expected shift. Also in this case, agreement can be easily achieved. To conclude, all the three models are marginally consistent with the data. No better comparison is possible at this stage.

\section{General discussion and concluding remarks}

We have presented a package of numerical codes to compute the spectroscopic and photometric properties of model galaxies by combining the evolutionary population synthesis technique with the 3D geometrical structure of the galaxy and its history of star formation and chemical enrichment, provided by NB-TSPH simulations. The tool is very flexible in the way input libraries of evolutionary tracks, isochrones, SSPs and important physical laws such as the initial mass function, star formation rate, and metallicity enrichment can be changed, tested, and added to the database for future use. Finally, it can be adapted to any photometric system currently in use. The method has been tested so far on three models of ETGs evolved within different cosmological backgrounds and the analysis has been done in the following photometric systems: Bessell-Brett, SDSS, COSMOS, and GOODS filters among all the ones we have at disposal. Our main results may be summarized as follows:

1. The application of the tool to three model galaxies at our disposal, allow us to compute the SED, magnitudes, and colors as function of time and redshift, together with the evolutionary and cosmological corrections. With the aid of it, we analyzed samples of ETGs taken from the COSMOS and GOODS databases, and made a qualitative and quantitative comparison between theoretical results and observational data. For both data-sets, we find that the simulated colors for the different cosmological scenarios follow the general trend over the entire range of redshift considered and are in good agreement with the data up to $z \sim 1$, above which the number of observed ETGs falls abruptly. In conclusion, within the redshift range considered, all the simulated colors reproduce quite well the observational data.

2. We have also generated synthetic $2 \mathrm{D}$ images of the galaxy models in a given photometric system. These synthetic images can be processed with the same algorithms used to analyze real images to derive the structural and morphological parameters, e.g. the galaxy's $R_{\mathrm{e}}$ and the luminosity within this, the shape indices through Fourier and Sérsic analysis, the color profiles, and the radial profiles of most of the parameters that define the structure of galaxies. We find that the luminosity profiles of the model galaxies at $z=0$ can be reasonably fitted with a Sérsic $R^{1 / n}$ law. The evaluation of the $R_{\mathrm{e}}$ in the photometric bands of SDSS shows the same dependence on the passband wavelength range of the observational data. Furthermore, the isophotes are well approximated by ellipses, with only a weak radial variation in position angle and ellipticity. Small but significant deviations from perfect ellipses are also measured. In general, we can recover properties that resemble those of observed galaxies.

3. In addition to that, we looked at the Kormendy relation, one of the Scaling Laws of ETGs, for which all theoretical counterparts of observational data were available. The theoretical luminosities and effective radii of the models are consistent with the archival data from the SDSS for a sample of ETGs.

From the above results we can conclude that the package provides good results that permit to reasonable recover the observational properties of ETGs. Owing to the small number of galaxy models to our disposal, neither statistical generalization of the results is possible, nor the underlying cosmological background can be tested. We have to wait for a more complete library of model galaxies in different cosmological scenarios to better elucidate the evolution of photometric properties and scaling laws, and to provide insight into the meaning of the correlations (or lack thereof) between shape, kinematics, and photometry of ETGs.

Acknowledgements. We like to thank Dr. Mariangela Bernardi for kindly providing the data from the SDSS database.

\section{References}

Aragon-Salamanca, A., Ellis, R. S., Couch, W. J., \& Carter, D. 1993, MNRAS, 262,764

Arimoto, N., \& Yoshii, Y. 1987, A\&A, 173, 23

Barger, A. J., Cowie, L. L., Smail, I., et al. 1999, AJ, 117, 2656

Barnes, J., \& Hut, P. 1986, Nature, 324, 446

Barnes, J. E., \& Hernquist, L. 1996, ApJ, 471, 115

Bell, E. F., Wolf, C., Meisenheimer, K., et al. 2004, ApJ, 608, 752

Bender, R. 1997, in The Nature of Elliptical Galaxies, ed. M. Arnaboldi, G. S. Da Costa, \& P. Saha, ASP Conf. Ser., 116, 11

Bender, R., Ziegler, B., \& Bruzual, G. 1996, ApJ, 463, L51

Bender, R., Saglia, R. P., Ziegler, B., et al. 1998, ApJ, 493, 529

Benítez, N. 2000, ApJ, 536, 571

Benz, W. 1990, in Numerical Modelling of Nonlinear Stellar Pulsations Problems and Prospects, ed. J. R. Buchler, 269

Berczik, P., Hensler, G., Theis, C., \& Spurzem, R. 2003, Ap\&SS, 284, 865

Bernardi, M., Sheth, R. K., Annis, J., et al. 2003a, AJ, 125, 1817

Bernardi, M., Sheth, R. K., Annis, J., et al. 2003b, AJ, 125, 1849

Bernardi, M., Sheth, R. K., Annis, J., et al. 2003c, AJ, 125, 1866

Bertelli, G., Bressan, A., Chiosi, C., Fagotto, F., \& Nasi, E. 1994, A\&AS, 106, 275

Bertin, E., \& Arnouts, S. 1996, A\&AS, 117, 393

Bessell, M. S. 1990, PASP, 102, 1181

Bessell, M. S., \& Brett, J. M. 1988, PASP, 100, 1134 
Bica, E., Barbuy, B., \& Ortolani, S. 1991, ApJ, 382, L15

Boissier, S., \& Prantzos, N. 1999a, Ap\&SS, 265, 409

Boissier, S. \& Prantzos, N. 1999b, MNRAS, 307, 857

Bower, R. G., Lucey, J. R., \& Ellis, R. S. 1992a, MNRAS, 254, 601

Bower, R. G., Lucey, J. R., \& Ellis, R. S. 1992b, MNRAS, 254, 589

Bressan, A., Chiosi, C., \& Fagotto, F. 1994, ApJS, 94, 63

Brinchmann, J., \& Ellis, R. S. 2000, ApJ, 536, L77

Bundy, K., Ellis, R. S., \& Conselice, C. J. 2005, ApJ, 625, 621

Bundy, K., Ellis, R. S., Conselice, C. J., et al. 2006, ApJ, 651, 120

Butcher, H., \& Oemler, Jr., A. 1978, ApJ, 219, 18

Capak, P., Aussel, H., Ajiki, M., et al. 2007, ApJS, 172, 99

Cardelli, J. A., Clayton, G. C., \& Mathis, J. S. 1989, ApJ, 345, 245

Carraro, G., Lia, C., \& Chiosi, C. 1998, MNRAS, 297, 1021

Castelli, F., Gratton, R., \& Kurucz, R. 1997, A\&A, 318, 841

Chiosi, C. 2000, in Spectro-photometric Dating of Stars and Galaxies, ed. I. Hubney, S. R. Heap, \& R. H. Cornett, ASP Conf. Ser, 192, 251

Chiosi, C., \& Carraro, G. 2002, MNRAS, 335, 335

Cimatti, A. 2009, in AIP Conf. Ser. 1111, ed. G. Giobbi, A. Tornambe, G. Raimondo, M. Limongi, L. A. Antonelli, N. Menci, \& E. Brocato, 191

de Vaucouleurs, G. 1948, Ann. Astrophys., 11, 247

Djorgovski, S. G., \& Davis, M. 1987, ApJ, 313, 59

Draine, B. T., \& Lee, H. M. 1984, ApJ, 285, 89

Dressler, A. 1980, ApJ, 236, 351

Dressler, A., Lynden-Bell, D., Burstein, D., et al. 1987, ApJ, 313, 42

Eggen, O. J., Lynden-Bell, D., \& Sandage, A. R. 1962, ApJ, 136, 748

Ellis, R. S., Smail, I., Dressler, A., et al. 1997, ApJ, 483, 582

Ferrini, F., \& Poggianti, B. M. 1993, ApJ, 410, 44

Fioc, M., \& Rocca-Volmerange, B. 1997, A\&A, 326, 950

Franx, M. 1995, in Stellar Populations, ed. P. C. van der Kruit, \& G. Gilmore, IAU Symp., 164, 269

Fukugita, M., Hogan, C. J., \& Peebles, P. J. E. 1998, ApJ, 503, 518

Fukugita, M., Ichikawa, T., Doi, M., Shimasaku, K., \& Schneider, D. 1996, AJ, 111,1748

Galletta, G., Casasola, V., Piovan, L., Merlin, E., \& Bettoni, D. 2007, A\&A, 462, 495

Giavalisco, M., Dickinson, M., Ferguson, H. C., et al. 2004, ApJ, 600, L103

Gibson, B. K. 1997, MNRAS, 290, 471

Girardi, L., Chiosi, C., Bertelli, G., \& Bressan, A. 1995, A\&A, 298, 87

Girardi, L., Bressan, A., Bertelli, G., \& Chiosi, C. 2000, A\&AS, 141, 371

Girardi, L., Bertelli, G., Bressan, A., et al. 2002, A\&A, 391, 195

Graham, A. W., Erwin, P., Trujillo, I., \& Asensio Ramos, A. 2003, AJ, 125, 2951

Grazian, A., Fontana, A., de Santis, C., et al. 2006, A\&A, 449, 951

Guiderdoni, B., \& Rocca-Volmerange, B. 1987, A\&A, 186, 1

Guiderdoni, B., \& Rocca-Volmerange, B. 1988, A\&AS, 74, 185

Harris, W. E. 1996, AJ, 112, 1487

Hogg, D. W. 1999 [arXiv: astro-ph/9812049], unpublished

Hubble, E. 1936, ApJ, 84, 517

Im, M., Griffiths, R. E., Ratnatunga, K. U., \& Sarajedini, V. L. 1996, ApJ, 461, L79

Immeli, A., Samland, M., Gerhard, O., \& Westera, P. 2004, A\&A, 413, 547

Jedrzejewski, R. I. 1987, MNRAS, 226, 747

Jorgensen, I., \& Hjorth, J. 1997, in Galaxy Scaling Relations: Origins, Evolution and Applications, ed. L. N. da Costa, \& A. Renzini, 175

Jorgensen, I., Franx, M., \& Kjaergaard, P. 1996, MNRAS, 280, 167

Kauffmann, G., White, S. D. M., \& Guiderdoni, B. 1993, MNRAS, 264, 201

Kobayashi, C. 2004a, Publications of the Astronomical Society of Australia, 21, 183

Kobayashi, C. 2004b, MNRAS, 347, 740

Kobayashi, C. 2005, MNRAS, 361, 1216

Kodama, T., \& Arimoto, N. 1997, A\&A, 320, 41

Kolb, E. W., \& Turner, M. S. 2000, Eur. Phys. J. C, 15, 125

Kurucz, R. 1993, in The Stellar Populations of Galaxies, ed. B. Barbuy, \& A. Renzini (Dordrecht: Kluwer), IAU Symp., 149, 255

La Barbera, F., Busarello, G., Merluzzi, P., Massarotti, M., \& Capaccioli, M. 2003, ApJ, 595, 127

Larson, R. B. 1975, MNRAS, 173, 671
Li, C. Jing, Y. P., Kauffmann, G., et al. 2006a, MNRAS, 368, 37 Li, C., Kauffmann, G., Jing, Y. P., et al. 2006b, MNRAS, 368, 21 Li, C., Kauffmann, G., Wang, L., et al. 2006c, MNRAS, 373, 457 Li, C., Jing, Y. P., Kauffmann, G., et al. 2007, MNRAS, 376, 984 Lia, C., Portinari, L., \& Carraro, G. 2002, MNRAS, 330, 821 Lucy, L. B. 1977, AJ, 82, 1013

Merlin, E., \& Chiosi, C. 2006, A\&A, 457, 437

Merlin, E., \& Chiosi, C. 2007, A\&A, 473, 733

Mobasher, B., Capak, P., Scoville, N. Z., et al. 2007, ApJS, 172, 117

Oke, J. B., \& Sandage, A. 1968, ApJ, 154, 21

Patton, D. R., Pritchet, C. J., Yee, H. K. C., Ellingson, E., \& Carlberg, R. G. 1997, ApJ, 475, 29

Peebles, P. 2002, in A New Era in Cosmology, ASP Conf. Ser., 283, 351

Pérez-González, P. G., Rieke, G. H., et al. 2008, ApJ, 675, 234

Piovan, L., Tantalo, R., \& Chiosi, C. 2003, A\&A, 408, 559

Piovan, L., Tantalo, R., \& Chiosi, C. 2006a, MNRAS, 366, 923

Piovan, L., Tantalo, R., \& Chiosi, C. 2006b, MNRAS, 370, 1454

Pipino, A., \& Matteucci, F. 2004, MNRAS, 347, 968

Rocca-Volmerange, B., \& Guiderdoni, B. 1988, A\&AS, 75, 93

Samland, M. 2001, in Dynamics of Star Clusters and the Milky Way, ed. S. Deiters, B. Fuchs, A. Just, R. Spurzem, \& R. Wielen, ASP Conf. Ser., 228, 559

Samland, M., \& Gerhard, O. E. 2000, in Liege International Astrophysical Colloquia, ed. A. Noels, P. Magain, D. Caro, E. Jehin, G. Parmentier, \& A. A. Thoul, 35, 415

Samland, M., Hensler, G., \& Theis, C. 1997, ApJ, 476, 544

Scannapieco, C., Tissera, P. B., White, S. D. M., \& Springel, V. 2006a, MNRAS, 371,1125

Scannapieco, E., Kawata, D., Brook, C. B., et al. 2006b, ApJ, 653, 285

Schade, D., Lilly, S. J., Crampton, D., et al. 1999, ApJ, 525, 31

Scoville, N., Aussel, H., Benson, A., et al. 2007, ApJS, 172, 150

Searle, L., Sargent, W. L. W., \& Bagnuolo, W. G. 1973, ApJ, 179, 427

Sérsic, J. L. 1968, Atlas de galaxias australes, Cordoba, Argentina, Observatorio Astronomico

Shimasaku, K., Fukugita, M., Doi, M., et al. 2001, AJ, 122, 1238

Spergel, D. N., Verde, L., Peiris, H. V., et al. 2003, ApJS, 148, 175

Steidel, C. C., Giavalisco, M., Dickinson, M., \& Adelberger, K. L. 1996, AJ, 112,352

Steidel, C. C., Adelberger, K. L., Giavalisco, M., Dickinson, M., \& Pettini, M. 1999, ApJ, 519, 1

Steidel, C. C., Adelberger, K. L., Shapley, A. E., et al. 2003, ApJ, 592, 728

Stoughton, C., Lupton, R. H., Bernardi, M., et al. 2002, AJ, 123, 485

Strateva, I., Ivezić, Ž., Knapp, G. R., et al. 2001, AJ, 122, 1861

Tantalo, R. 2005, in The Initial Mass Function 50 Years Later, ed. E. Corbelli, F. Palla, \& H. Zinnecker, ASSL, 327, 235

Tantalo, R., Chiosi, C., Bressan, A., \& Fagotto, F. 1996, A\&A, 311, 361

Tantalo, R., Chiosi, C., \& Bressan, A. 1998a, A\&A, 333, 419

Tantalo, R., Chiosi, C., Bressan, A., Marigo, P., \& Portinari, L. 1998b, A\&A, 335,823

Theis, C., Burkert, A., \& Hensler, G. 1992, A\&A, 265, 465

Treu, T., Ellis, R. S., Liao, T. X., et al. 2005, ApJ, 633, 174

Trujillo, I., Graham, A. W., \& Caon, N. 2001, MNRAS, 326, 869

Trujillo, I., Erwin, P., Asensio Ramos, A., \& Graham, A. W. 2004, AJ, 127, 1917

van der Wel, A., Franx, M., van Dokkum, P. G., et al. 2005, ApJ, 631, 145

van Dokkum, P. G., \& Franx, M. 1996, MNRAS, 281, 985

van Dokkum, P. G., Franx, M., Kelson, D. D., \& Illingworth, G. D. 1998, ApJ, 504, L17

van Dokkum, P. G., Franx, M., Fabricant, D., Illingworth, G. D., \& Kelson, D. D. 2000, ApJ, 541, 95

Vazdekis, A., Peletier, R. F., Beckman, J. E., \& Casuso, E. 1997, ApJS, 111, 203

Weinberg, S. 1972, Gravitation and Cosmology: Principles and Applications of the General Theory of Relativity

White, S. D. M., \& Rees, M. J. 1978, MNRAS, 183, 341

Worthey, G., Faber, S. M., González, J. J., \& Burstein, D. 1994, ApJS, 94, 687

York, D. G., Adelman, J., Anderson, Jr., J. E., et al. 2000, AJ, 120, 1579

Ziegler, B. L., \& Bender, R. 1997, MNRAS, 291, 527 